
allemande

47-2 | 2015

Retour sur le modèle Rhénan : humanisme, capitalisme et métropolisation | Patrimonialisation du passé « allemand » en Europe centrale après 1990

\title{
Der Oberrhein, Fiktion und Fabrikation eines Metropolraums
}

\section{Volker Ziegler}

\section{(2) OpenEdition \\ Journals}

Édition électronique

URL : https://journals.openedition.org/allemagne/292

DOI : 10.4000/allemagne.292

ISSN : 2605-7913

Éditeur

Société d'études allemandes

Édition imprimée

Date de publication : 16 décembre 2015

Pagination : 321-344

ISSN : 0035-0974

Référence électronique

Volker Ziegler, „Der Oberrhein, Fiktion und Fabrikation eines Metropolraums“, Revue d'Allemagne et des pays de langue allemande [Online], 47-2 | 2015, Online erschienen am: 13 Dezember 2017, abgerufen am 19 Mai 2021. URL: http://journals.openedition.org/allemagne/292 ; DOI: https://doi.org/10.4000/ allemagne.292 


\section{Der Oberrhein, Fiktion und Fabrikation eines Metropolraums}

- Volker Ziegler*

Die Städte und Gebiete am Oberrhein sind seit jeher Spielball der Beziehungen zwischen Frankreich und Deutschland, aber auch zwischen der Schweiz und diesen beiden Ländern. Lange Zeit behinderte das schwierige Erbe der beiden Weltkriege eine übergreifende Betrachtung. Erst im Vorfeld der Vollendung des Europäischen Binnenmarktes zum 1. Januar 1993 wurde die Frage nach der Identität und den Entwicklungsperspektiven der Region im Herzen Europas neu gestellt und mit anderen Regionen Europas in Zusammenhang gebracht.

So auch bei der 17. Mailänder Triennale, die sich 1988

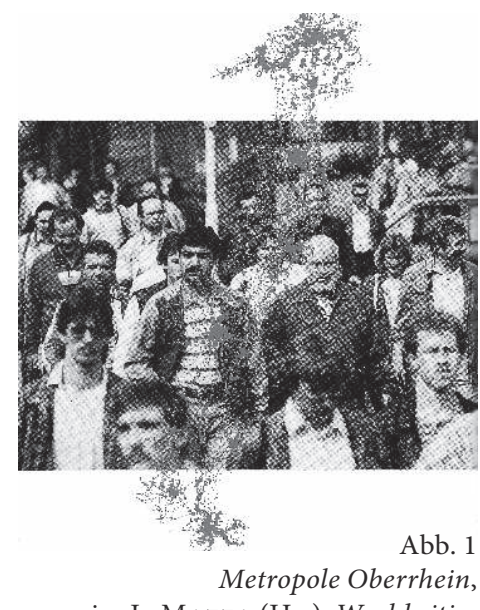

in: L. Mazza (Hg.), World cities

(Anm. 1), S. 200. Die Städte der Welt und die Zukunft der Metropolen zum Thema machte ${ }^{(1)}$. Vierzehn Staaten sowie die Europäische Gemeinschaft und die Vereinten Nationen beschäftigten sich mit der Zukunft der Metropolen, wobei die meisten Länder ihre jeweilige Hauptstadt präsentierten. Auch die Europäische Gemeinschaft betonte die zentrale Bedeutung der „großen Großstädte“. Ihre „Hub-Funktion“ und ihre internationalen Vernetzungen würden sie zu Hebelpunkten für die Schaffung eines vereinten europäischen Wirtschaftsraums machen, der dank Hochgeschwindigkeitszügen, modernen Flughäfen, neuen Übertragungstechnologien und Mobilitätsprogrammen (Erasmus) großräumig verschmelzen soll. Von einer Neudeutung des Metropolenbegriffs war von dieser Seite her kaum etwas zu hören oder zu sehen (Abb. 1).

* Maître-assistant à l'École nationale supérieure d'architecture de Strasbourg (ENSAS), co-responsable du double master „Architecture et projet urbain dans les eurorégions“ cohabilité avec le Karlsruher Institut für Technologie (KIT).

1 Luigi Mazza (Hg.), World cities and the future of the metropoles. International participations, Ausst.Kat. der 17. Mailänder Triennale 1988, Bd. 2, Mailand, Electa, 1988. 


\section{Alternative Metropolräume für ein Europa der Regionen}

Drei Beiträge stachen jedoch aus diesem Gleichklang hervor. So konnten die Besucher in der Ausstellung im Mailänder Palazzo dell'Arte über ein mehr als vierzig Meter langes hinterleuchtetes Luftbild der Po-Ebene laufen, deren verstädterte Landschaft in Kontrast zur „schönen alten Stadt“ mit ihren identitätsstiftenden Stadträumen und Kollektivbauten gesetzt wurde. Die Frage nach der Identität der Po-Ebene, die als metropolitaner Raum begriffen wurde, konnte 1988 immerhin gestellt werden und wurde seither vor allem durch die am Istituto Universitario di Architettura in Venedig (IUAV) durchgeführten Untersuchungen von Francesco Indovina und später von Bernardo Secchi auch in ihren Qualitäten gewürdigt. Indovina prägte den Begriff der città diffusa (diffuse Stadt) und meinte damit nicht etwa die Fragmente einer teppichartigen Patchwork-Metropole ${ }^{(2)}$. Laut Secchi ähneln die Siedlungsstrukturen der Po-Ebene eher der fein verästelten, durchlässigen Struktur eines Schwamms, in dem sich die Bewohner zwischen ihren Alltagsaktivitäten hin- und herbewegen und dadurch ihre eigene, persönliche Stadt formen ${ }^{(3)}$. Für

Fachleute und Entscheidungsträger impliziert dies aber ein Umdenken: eine Region wie das Veneto soll nicht mehr aus dem Blickwinkel der historischen Zentralstädte als deren verstädtertes Umland gesehen werden - das heißt konkret als Ausdehnungsoder Einflussgebiet von Venedig, Padua und Treviso - sondern als ein von alters her dicht besiedelter Raum, dessen ungeplante Urbanisierung auf den Unternehmergeist eines ortsansässigen, prosperierenden Mittelstands zurückzuführen ist. Seit den neunziger Jahren wird das Schlagwort città diffusa auch in der italienischsprachigen Schweiz verwendet, um die Siedlungsstruktur der mit ineinander verwachsenen Städten und Gemeinden dicht bebauten Täler des Tessins zu beschreiben, das über die Staatsgrenze hinweg immer mehr mit dem Großraum Mailand verschmilzt ${ }^{(4)}$ (Abb. 2).

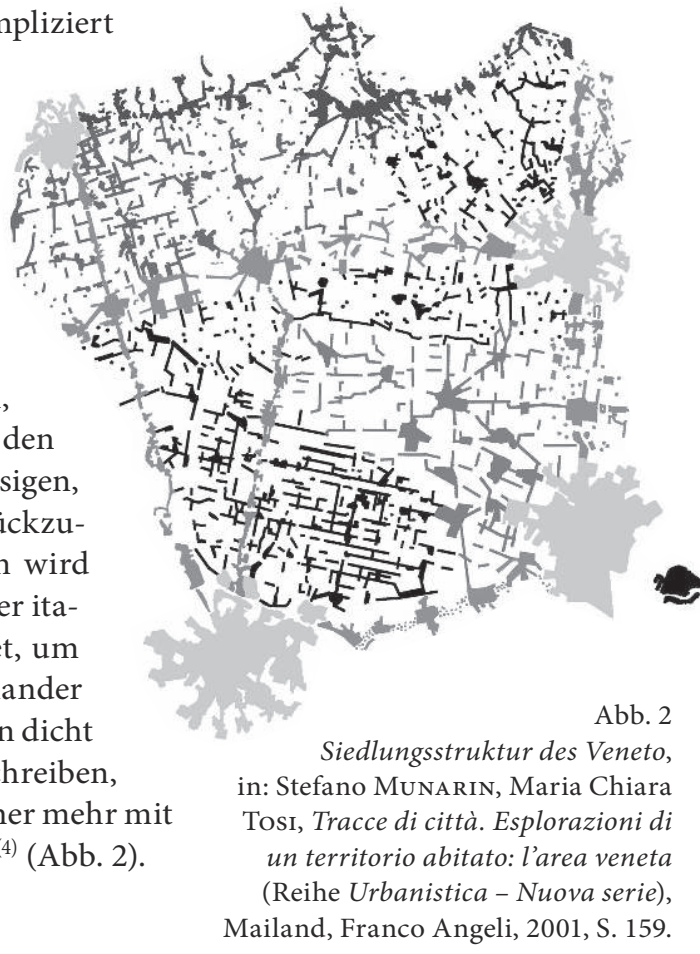

2 Francesco Indovina (Hg.), La città diffusa, Venedig, Istituto Universitario di Architettura di Venezia, Dipartimento di analisi economica e sociale del territorio, 1990.

3 „Comment agir sur la ,città diffusa?"“ (Bericht über den Vortrag von Bernardo Secchi), 5 à 7 (Vortragsreihe des Club Ville Aménagement unter der Leitung von Ariella Masboungi), April 2002, unter http:// www.club-ville-amenagement.org/_upload/ressources/productions/5a7/2002/5a7_cva_cittadiffusa_ verbatim.pdf (abgerufen am 20.6.2015).

4 Jan CAPOL, „La città diffusa: das Tessin als Stadt“, Hochparterre, Nr. 6-7 (1996), S. 18-23. 
In ihrem Beitrag zur Triennale beschäftigten sich die Niederlande mit der Entwicklung der holländischen Randstad als ein von Rotterdam über Den Haag bis Amsterdam und Utrecht zusammenwachsender Ring von Städten, der sich um einen großen, zusammenhängenden Landschafts- und Naherholungsraum legt. In der 1988 als Metropole im Werden begriffenen Randstad wird nicht nur der Blickwinkel, sondern auch die Position von Stadt zu Land vertauscht, denn im Zentrum der RandstadMetropole schlägt Hollands groene hart (grünes Herz). So wie das unscharfe Bild der oberitalienischen Metropole durch die Untersuchungen am IAUV zur città diffusa des Veneto an Klarheit gewann, so füllte sich auch das abstrakte Bild der Randstad durch das 1987 von der niederländischen Regierung angestoßene Forschungsprogramm Stedelijke netwerken (Städtenetze), in das die Universitäten Amsterdam, Utrecht und Delft eingebunden wurden, mit Leben. In die niederländische Vorstellung von Stadt als Netzwerk fließen auch die seit den siebziger Jahren verfolgten Überlegungen des französischen Geografen und Raumplaners Gabriel Dupuy ein ${ }^{(5)}$. Ausgehend von der Beobachtung, dass Infrastrukturnetze eine Vielzahl an möglichen Verbindungen erlauben, auf die sich urbane Lebensweisen stützen können, stellt Dupuy dem Planungsfunktionalismus der Trente Glorieuses eine Raumordnung entgegen, die das flächendeckende zoning durch das Konzept eines urbanisme des réseaux ersetzt ${ }^{(6)}$.

Ähnlich wie die oberitalienische Metropolregion wird die Randstad nicht als ein polyzentrischer Metropolraum betrachtet, der im Zuge der Verstädterung und Aktivitätsverlagerung im Umland einer monozentrischen Kernstadt entstanden ist, sondern vielmehr als eine polyzentrische Städteregion, deren inter-urbane Strukturen sich „aus einer zunehmenden funktionalen Verflechtung mehrerer Kernstädte in räumlicher Nähe“ entwickelt haben ${ }^{(7)}$. Die Untersuchungen mündeten 1993 in das staatliche VINEX-Wohnungsbauprogramm, das sich zum Ziel nahm, bis 2015 landesweit 750.000 preisgünstige Wohnungen zu bauen und diese in sozial tragfähigen und gut angebunden Neubaugebieten nahe bestehender Stadtzentren zu konzentrieren. Damit sollte eine großräumige Mobilität zwischen Wohn-, Arbeits- und Freizeitstätten möglichst verhindert sowie Landschaftsräume und Wasserlagen besser geschützt werden. Insbesondere die mittelgroßen Städte in der Randstad wurden durch das VINEX-Programm gefördert und über ein engmaschiges öffentliches Verkehrsnetz miteinander verbunden. Mit dem Zweitnamen „Deltametropole“ unterstreicht die Randstad seit Ende der neunziger Jahre ihren metropolitanen Anspruch (Abb. $3+4$ ).

5 Ina KlAASEN, „Network Cities: Operationalising a Strong but Confusing Concept“, ENHR (European Network for Housing Research), Tagungsbericht der Internationalen Konferenz Sustainable Urban Areas, Rotterdam, 25-28.6.2007, unter https://www.academia.edu/278932/Network_Cities_ Operationalising_a_Strong_but_Confusing_Concept (abgerufen am 20.6.2015).

6 Gabriel Dupuy, L’urbanisme des réseaux, théories et méthodes, Paris, Armand Colin (Reihe Géographie), 1991.

7 Kati Volgmann, „Neue Kerne in metropolitanen Räumen - Polyzentrische Strukturen und Funktionen im Metropolraum Rhein-Ruhr“, in: Anna Growe u. a. (Hg.), Polyzentrale Stadtregionen - Die Region als planerischer Handlungsraum (Arbeitsberichte der Akademie für Raumforschung und Landesplanung, 3), S. 203-216, hier S. 207. 


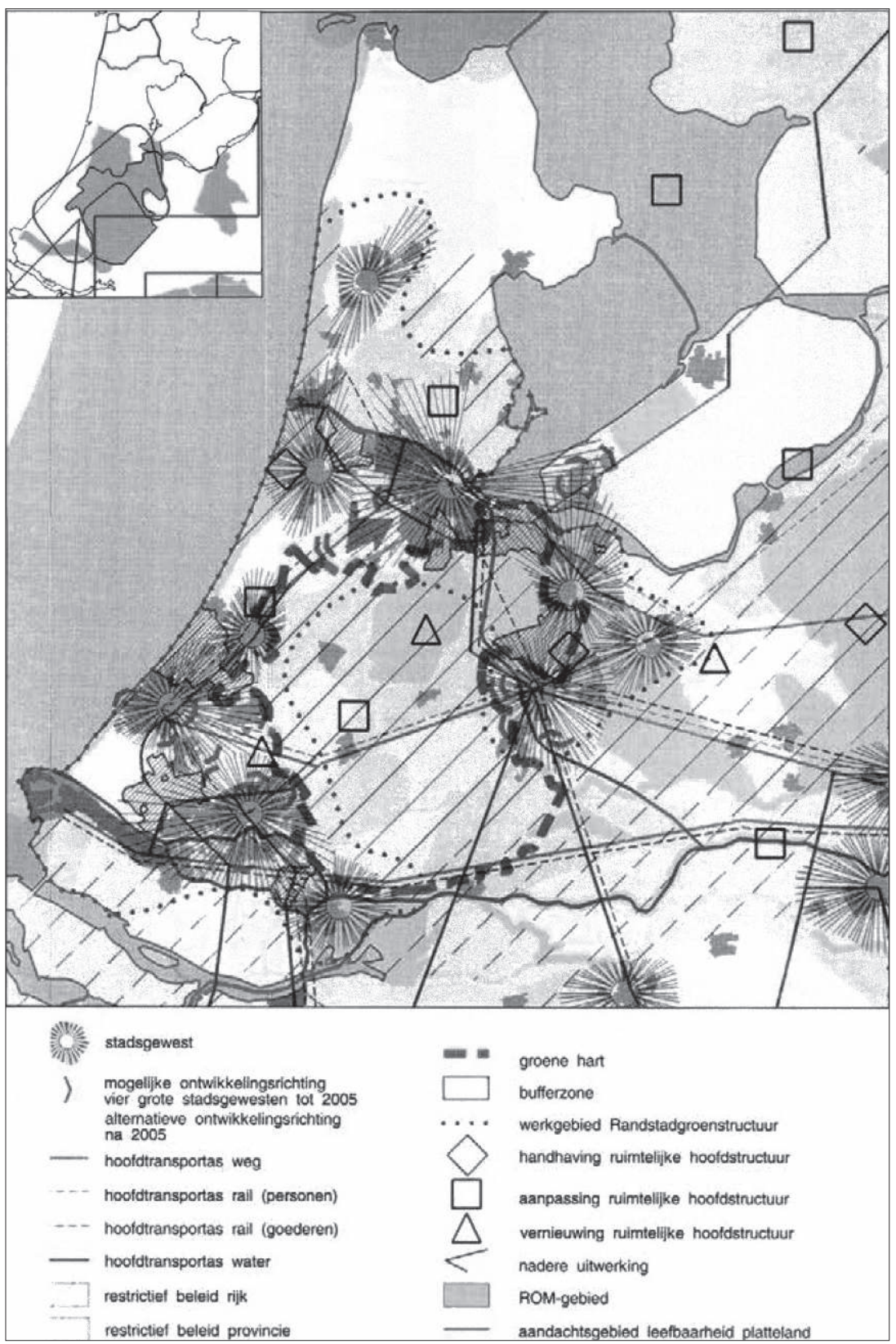

Abb. 3 - Räumliche Entwicklungsperspektive der Randstad,

in: VINEX (Vierde nota over de ruimtelijke ordening Extra), Teil 3: Kabinetsstandpunt, Tweede Kamer der Staten-Generaal, 21879, Nr. 5-6 (Versammlungsjahr 1990-1991), S. 199. 


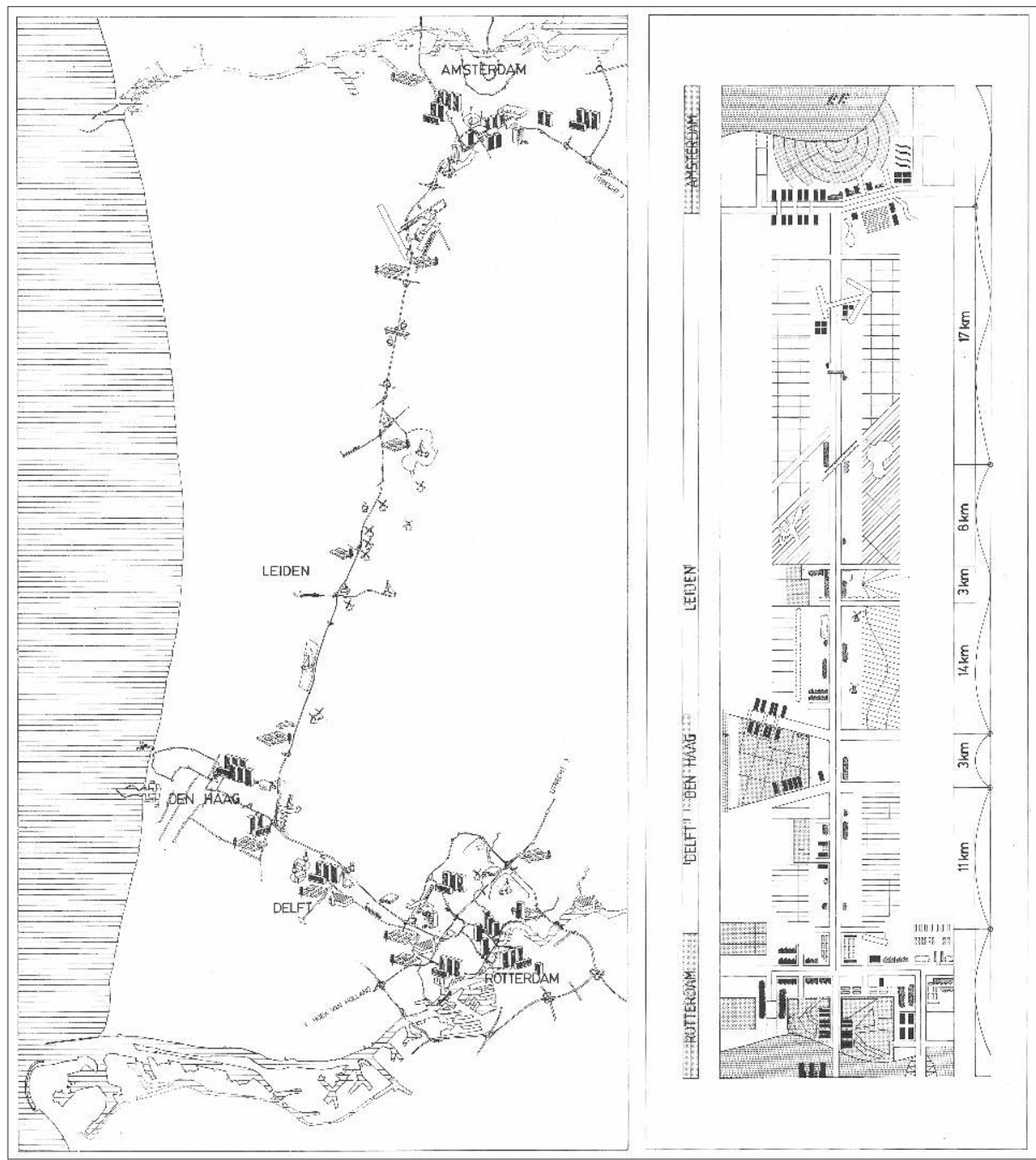

Abb. 4 - Zweimal Randstad von der Autobahn,

Zeichnungen von K. Fibbe u. M. Versteeg, in: L. MazzA (Hg.), World cities (Anm. 1), S. 164.

Auch die Arbeitsgruppe um den Karlsruher Stadtplaner Martin Einsele präsentierte in Mailand einen differenzierten Metropolenraum. Der Oberrhein - eine „andere Metropole“, so der Titel des vielbeachteten dreisprachigen Beitrags des Landes Baden-Württemberg, wurde dabei als Region dargestellt, in der die wichtigsten Agglomerationen zusammen mit dem Geflecht kleinerer Städte eine vielgestaltige „Städte-Landschaft“ mit dezentraler Siedlungsstruktur bei gleichzeitig hoher Bevölkerungsdichte bilden ${ }^{(8)}$.

8 Martin Einsele (Hg.), Le Rhin supérieur - une „métropole alternative“. Der Oberrhein - eine „andere Metropole“. The Upper Rhine - an „Alternative Metropolis“, Ausst.-Kat. des Beitrags für die 17. Mailänder Triennale 1988, Karlsruhe, Arbeitsgruppe Triennale Baden-Württemberg, 1989 (2. Aufl.). 
Nach Mailand wurde die Ausstellung 1989-1990 in zehn italienischen, französischen und deutschen Städten gezeigt und die Arbeitsgruppe Einsele besonders in Frankreich zu großen städtebaulichen Wettbewerben eingeladen (Strasbourg-Kehl, 1990-1991; Paris-Mission Grand Axe, 1991) ${ }^{(9)}$.

Im Rahmen einer Studie zum raumordnerischen Orientierungsrahmen für das Gebiet der Oberrheinkonferenz setzte Einsele als Leiter einer deutsch-französischschweizerischen Arbeitsgruppe seine Überlegungen zur „Metropole Oberrhein“ in den neunziger Jahren fort. Der Ausnahmecharakter dieser zweisprachig vorliegenden, 1999 unter dem deutschen Titel Lebensraum Oberrhein... eine gemeinsame Zukunft veröffentlichten Untersuchung liegt in der Tatsache, dass sich Akteure aus allen drei Ländern des Oberrheingebiets gemeinsam und tiefgreifend mit einem Raum befassten, der zwar im Zentrum Europas liegt, aber jeweils am Rande dieser drei Länder ${ }^{(10)}$. Die Studie entwarf Entwicklungsszenarien für eine „dezentrale Dreiländer-Metropole Oberrhein“, die als „Städtenetz in einer Gartenlandschaft“ aufgefasst wird, mit dem Rhein als Rückgrat eines metropolitanen Regionalparks. Gleichzeitig sollen wirtschafts- und verkehrspolitische Maßnahmen greifen, um das Rheintal zum „Technology Valley“ auszubauen und Verkehrsinfrastrukturen so zu vernetzen, dass die Verbindung der großen Städte untereinander und die Erreichbarkeit bedeutender regionaler Einrichtungen von überall her innerhalb einer Stunde bewerkstelligt werden kann (Abb. 5).

Dabei betont die Studie, dass der heutige politische Oberrhein Teil eines geografisch und historisch weiter zu fassenden Oberrheingebiets zwischen Basel und Mainz ist. Im Vorwort beschreibt ihn der Straßburger Historiker Michel Hau als eine durch ihre hohe Bevölkerungsdichte geprägte „Zivilisation“, die im kurzlebigen Lothringer Mittelreich wurzelt ${ }^{(11)}$. Die von Frankreich und Deutschland jahrhundertelang umworbenen und umkämpften Gebiete dieses Zwischenreichs im Herzen Westeuropas erstreckten sich von den Niederlanden bis nach Italien; somit umfassen sie auch alle drei auf der Mailänder Triennale präsentierten Metropolregionen.

Für Hau fußt die große Dynamik und der relative Reichtum dieser Regionen gerade auf ihrer Zwischenstellung. Die Randlage zu Deutschland und zu Frankreich erlaubte es ihren kleinen Fürstentümern und Städten, gegenüber Kaiser oder König, Berlin, Wien oder Paris relativ unabhängig zu bleiben. Da die Politik der Zentralisierung erst sehr spät griff, konnte sich gegenüber der schwachen Obrigkeit ein auf Sparsamkeit und Solidarität fußender, bürgerlich geprägter Kapitalismus herausbilden, der in einem von häufigen wirtschaftlichen und politischen Schwankungen geprägten

9 Ute Langendörfer, Michael Peterek (Red.), Planungsraum - Lebensraum: Martin Einsele, Positionen 1949-1998, Karlsruhe, Universität Karlsruhe (Karlsruher Städtebauliche Schriften, 9), 1998, hier S. 145-169.

10 Deutsch-Französisch-Schweizerische Oberrheinkonferenz (Hg.), Gruppe ECORHS (ADEUS, ADAUHR, Martin Einsele, Gresco Conseils) (Red.), Lebensraum Oberrhein... eine gemeinsame Zukunft. Raumordnung für eine nachhaltige Entwicklung ohne Grenzen. Lire et construire l'espace du Rhin supérieur. Atlas transfrontalier pour aménager un territoire commun, Karlsruhe, G. Braun u. Straßburg, La Nuée Bleue, 1999.

11 Michel Hau, „Avant-propos. Un fleuve et des hommes. Vorwort. Der Oberrhein - eine Geschichte für sich“, ebd., S. 11-17. 


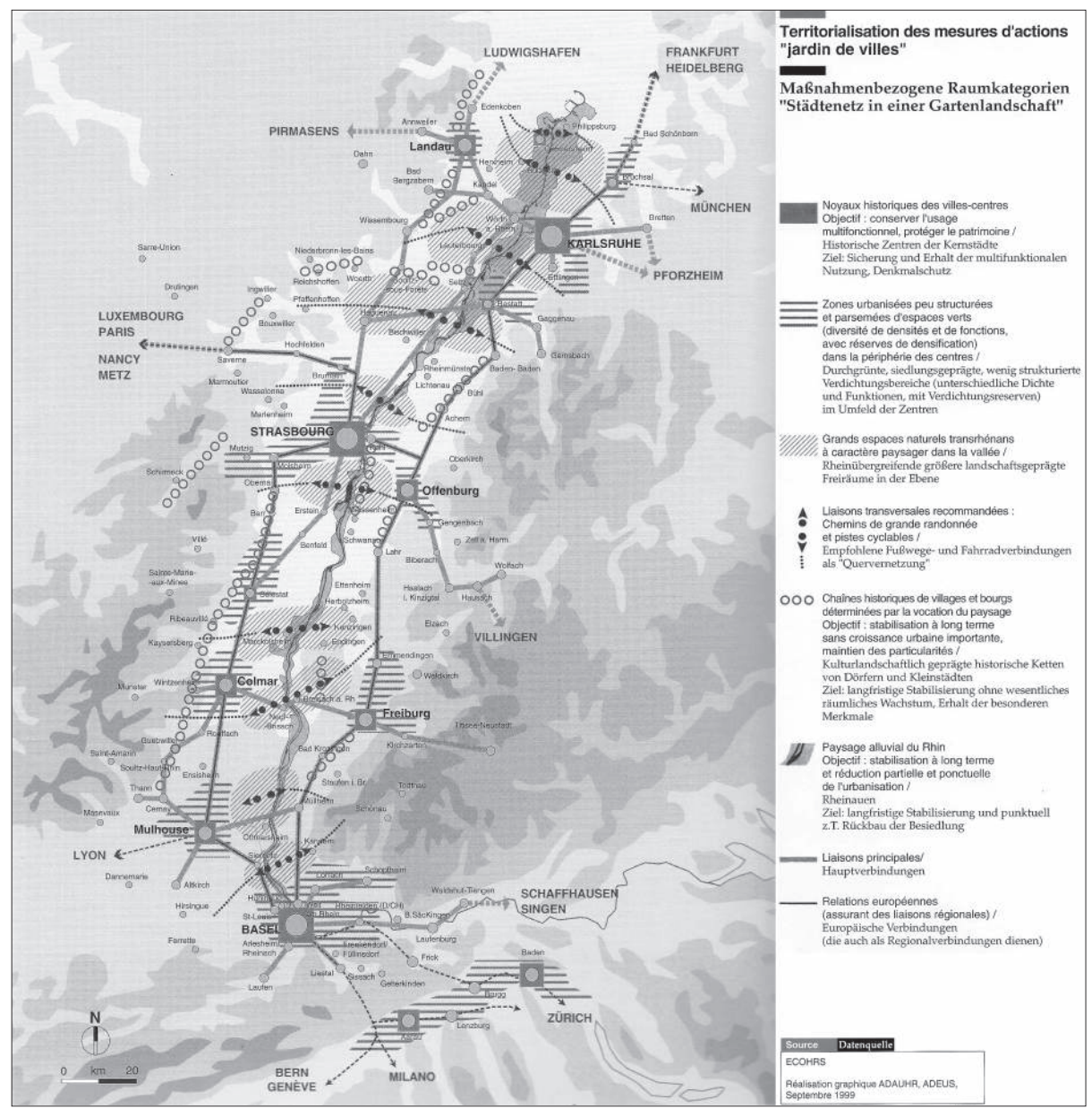

Abb. 5 - Metropole Oberrhein als Städtenetz in einer Gartenlandschaft,

in: Dt.-frz.-schw. Oberrheinkonferenz (Hg.), Lebensraum Oberrhein (Anm. 10), S. 146-147.

Kontext nicht auf kurzfristigen Gewinn setzte, sondern in langfristige Ziele und Projekte investierte. In Frankreich wie in Deutschland wird dieses Wirtschaftsmodell als „rheinischer Kapitalismus“ beschrieben.

Für die Entwicklung des Oberrheins war er quasi das Schmieröl, das sich entlang des Rheins und seiner Nebenflüsse in einem Netz von selbstverwalteten Städten und freien Reichsstädten verteilte. Der Fluss bildete schon früh die Lebensader eines Wirtschaftsraums für das aufstrebende Bürgertum in den Städten von Rotterdam bis Basel, mit ihren Handelsverbindungen zur Hanse und zu den unabhängigen Städten der Eidgenossenschaft, Flanderns und Norditaliens. Gleichermaßen einte der Rhein mit der Verbreitung des Humanismus und der Buchdruckkunst einen Kulturraum, der ab dem 19. Jahrhundert nach und nach zu einem Nord- und Südeuropa verbindenden Verkehrsraum wurde. Von der Regulierung und Anpassung des Rheins für die moderne Schifffahrt nach den Plänen Johann Gottfried Tullas über die ersten, 
privatwirtschaftlich initiierten Autostraßenprojekte des frühen 20. Jahrhunderts bis hin zu den Hochgeschwindigkeitstrassen unserer Zeit wurden und werden eine Vielzahl an Infrastrukturgroßprojekten verwirklicht, die mit massiven Umwelteingriffen einhergehen und die Kulturlandschaft am Oberrhein bedrohen ${ }^{(12)}$. Mit der Frage der Nachhaltigkeit all dieser Maßnahmen werden seit einigen Jahren jedoch andere Prioritäten gesetzt, und diese Perspektive macht sich auch die Studie der Oberrheinkonferenz zu eignen, indem sie den Oberrhein vor allem als „Lebensraum“ begreift. Gestern noch Industriekorridor, chaussée industrielle einer nach Aufschwung strebenden Nachkriegsgeneration, soll der Rhein nunmehr ein gemeinsam zu schützendes Landschaftssystem und ein Referenzraum für eine grenzüberschreitende Zusammenarbeit sein.

Die Studie arbeitet institutionelle, demografische und wirtschaftliche Gemeinsamkeiten und Unterschiede einer dicht besiedelten und politisch dezentral organisierten Region heraus, mit entsprechenden Auswirkungen auf Verhaltens- und Lebensweisen, auf Siedlungsstrukturen und Verstädterungsprozesse, auf kulturelle Ausprägungen oder Umweltrisiken. Die Besonderheit der „Städte-Landschaft“ liegt darin, dass keine der fünf wichtigsten Städte (Basel, Freiburg, Karlsruhe, Mulhouse, Straßburg) eine dominierende Rolle spielt. Vielmehr ergänzen sich diese zu einem Städtenetz, in das in Nord-Süd-Richtung eine dichte Folge von kleinen und mittelgroßen Städten beiderseits des Rheins eingebunden ist. Die Siedlungsstruktur wird dabei weitgehend durch die Topografie und die parallel zum Fluss verlaufenden großen Verkehrsinfrastrukturen konditioniert und bildet entlang der historischen Verkehrswege fast durchgängige Siedlungsbänder. Größere Siedlungsgebiete entstanden an den Kreuzungen mit den relativ schwachen Ost-West-Verbindungen, etwa am Austritt der Vogesen- und Schwarzwaldtäler oder am Rhein. Im Kontrast zu diesen urbanen Siedlungsräumen stehen die ländlichen Räume der Bergregionen und viele noch intakte Flusslandschaften des Oberrheins.

Die lineare Siedlungsentwicklung birgt für die Qualität der Landschafts- und Agrarwirtschaftsräume ein großes Risiko. Ähnlich wie das niederländische VINEXProgramm empfiehlt die Studie daher, die Entwicklung kleinerer und mittlerer Städte zu stärken. Ganz im Sinne des Leitbilds der „Stadt der kurzen Wege“ soll das städtische Wachstum verstärkt über ein verbessertes Management bestehender Siedlungsflächen auf kommunaler und interkommunaler Ebene, über den Ausbau des ÖPNV und allgemein über eine bessere Verknüpfung von Arbeit und Wohnen gesteuert werden. Zudem erscheint die Schaffung neuer oder Stärkung bestehender Ost-West-Transversalen in vielerlei Hinsicht als sehr wünschenswert, wobei die Studie nicht explizit auf die Folgen etwa in Bezug auf Standortattraktivität oder Entwicklung der Bodenpreise links und rechts des Rheins eingeht. Für die „dezentrale Dreiländer-Metropole Oberrhein“ werden drei Entwicklungsszenarien skizziert: die „Fortsetzung der Desurbanisierung“ (Urban Sprawl), die „Reurbanisierung (Dezentrale Konzentration)“ und die

12 Die unter den Folgen des Versailler Vertrags besonders stark betroffenen deutschen Städte und Konzerne am Oberrhein waren an der Schaffung einer modernen Straßenverbindung zwischen den Häfen der Nordsee und des Mittelmeers interessiert. 1926 wurde mit schweizerischer und italienischer Beteiligung der HaFraBa-Verein gegründet, der den Bau einer Autobahn von Hamburg über Frankfurt und Basel bis nach Genua durchsetzen wollte. S. Volker ZiEgLeR, „Les autoroutes du III ${ }^{e}$ Reich et leurs origines“, in: Jean-Louis CoHEn, Les années 30. L'architecture et les arts de l'espace entre industrie et nostalgie, Ausst.-Kat., Paris, Éd. du Patrimoine, 1997, S. 207-213. 
„nachhaltige Stadtlandschaft“(13). Letztere wird als wünschenswert erachtet und soll gerade in grenzüberschreitenden Planungen Leitbildcharakter für die Qualität von Stadtform und Architektur haben (Abb. 6).

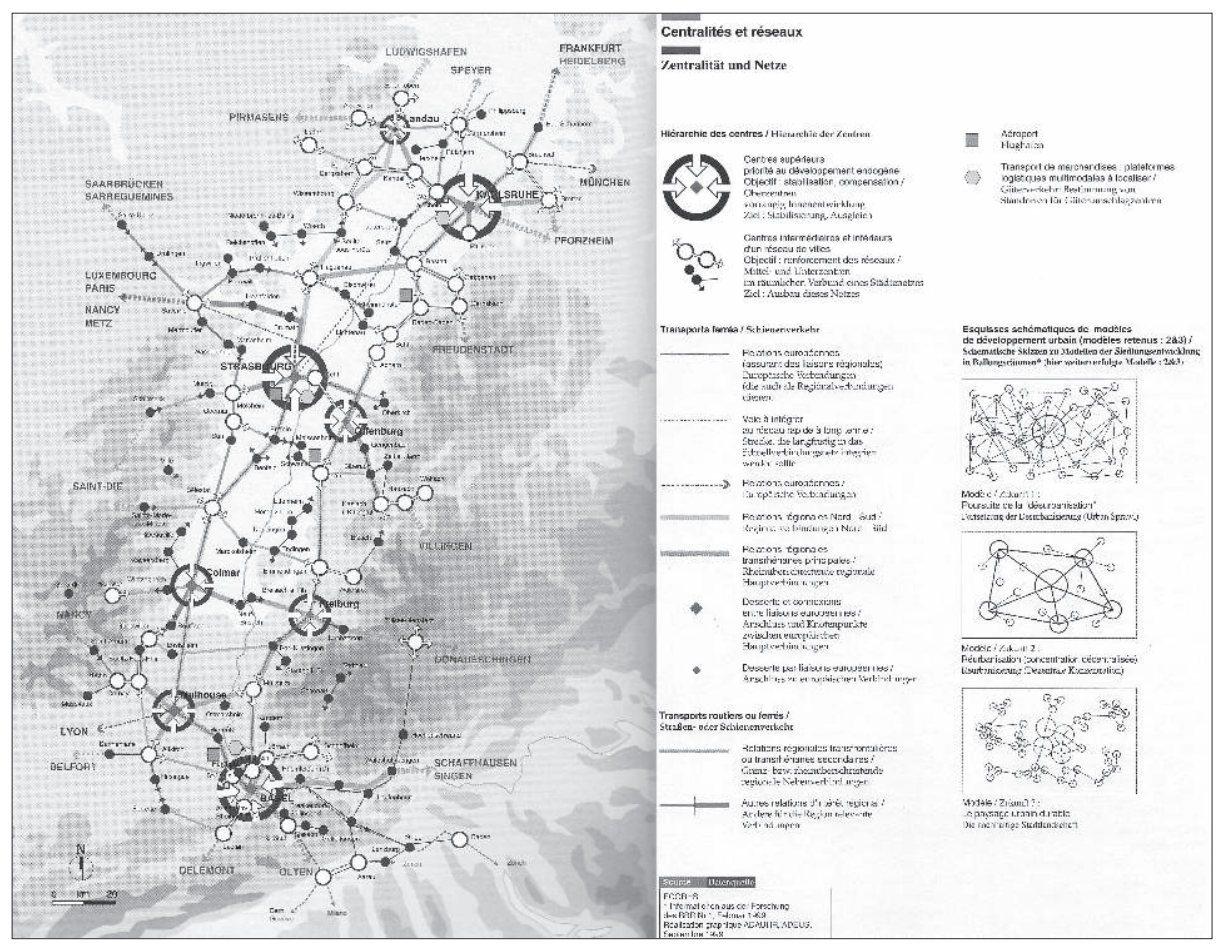

Abb. 6 - Dezentrale Dreiländer-Metropole Oberrhein,

in: Dt.-frz.-schw. Oberrheinkonferenz (Hg.), Lebensraum Oberrhein (Anm. 10), S. 140-141.

\section{Die andere Metropole und die Metropole der Anderen}

Das Postulat einer „anderen Metropole“ Oberrhein von 1988 lässt bewusst offen, worin diese Andersartigkeit genau bestehen könnte. Letztendlich muss sie ein vieldeutiges, aber damit auch unklares Konstrukt bleiben, um divergierende, ja „alternative“ - so die englischsprachige Bezeichnung der „Metropole Oberrhein“ - Vorstellungen beispielsweise zu ihrer Beschaffenheit, Erfahrbarkeit oder Weiterentwicklung unter einen Hut zu bringen $^{(14)}$. So firmiert die zweisprachige Nachfolgearbeit von 1999 als deutsch-französischschweizerische Gemeinschaftsproduktion unter zwei völlig verschiedenen Titeln, die jeweils andere Inhalte und Ziele der gemeinsamen Studie beleuchten: Lebensraum Oberrhein... eine gemeinsame Zukunft. Raumordnung für eine nachhaltige Entwicklung ohne Grenzen lautet der deutsche Titel, Lire et construire l'espace du Rhin supérieur. Atlas transfrontalier pour aménager un territoire commun der französische ${ }^{(15)}$.

13 Dt.-frz.-schw. Oberrheinkonferenz (Hg.), Lebensraum Oberrhein (Anm. 10), S. 141.

14 M. Einsele (Hg.), Le Rhin supérieur (Anm. 8).

15 Dt.-frz.-schw. Oberrheinkonferenz (Hg.), Lebensraum Oberrhein (Anm. 10). 
In den deutsch- und in den französischsprachigen, aber auch in den miteinander geführten Debatten zur Zukunft des Oberrheins verhält es sich oft ähnlich. Zum gleichen Thema tauchen immer wieder Begriffe auf, die zwar in die Sprache des Anderen übersetzt werden, aber beim Transfer in einen anderen kulturellen Erfahrungsraum gesetzt und entsprechend umgedeutet werden müssen. Zwei Begriffe, die besonders oft bemüht werden, sind auf deutscher Seite die „Städte-Landschaft“ und auf französischer Seite das modèle rhénan.

Mag der von der Arbeitsgruppe um Martin Einsele verwendete Begriff der "StädteLandschaft" einen französischen Leser wohl etwas verwundern, so hat er für den deutschen Leser neben der kulturgeschichtlichen auch eine planungsgeschichtliche Konnotation. Denn die Auflösung des Gegensatzes zwischen (historischer) Stadt und Land(schaft) wurde von deutschen Stadtplanern ab den dreißiger Jahren immer wieder hinterfragt. Sie avancierte im Konzept der „Stadtlandschaft“ zum Leitbild für den Wiederaufbau der kriegszerstörten Städte und diente bis in die sechziger Jahren als Begründung verschiedenartigster Planungen von Architekten und Stadtplanern mit unterschiedlichstem weltanschaulichen Hintergrund. Als gemeinsamer Nenner ist hierbei immer eine weiträumig in Nachbarschaften mit eher geringer Bebauungsdichte aufgelöste Großstadt gemeint. Wegweisende Stadtlandschaftskonzepte wurden von Konstanty Gutschow, Hans Bernhard Reichow oder Hans Scharoun entwickelt, aber auch von Planern, die in Gebieten tätig waren, wo sich die deutsche mit der französischen Einflusssphäre überschnitt. So entwarf der in Straßburg geborene Rudolf Schwarz im deutsch besetzten Lothringen und später als Generalplaner der Stadt Köln beispielhafte Siedlungspläne für ganze Stadtregionen (Stadtlandschaft Diedenhofen/Thionville, 1942-43; Stadtlandschaft Köln, 1949). Manche am Oberrhein tätigen Architekten und Stadtplaner verbanden in theoretischen Untersuchungen, aber auch in konkreten Planungen sehr früh Fragen der Stadt-Land-Beziehung, der Metropolentwicklung und der Regionalplanung, die sie gerade wegen der wechselhaften politischen Großwetterlage als wichtige Arbeitsfelder begriffen, und formten so das Arbeitsfeld des Raumplaners.

Eine Schlüsselfigur war der Bruchsaler Architekt, Stadtökonom und Städtebautheoretiker Roman Heiligenthal. Nach Stationen im Essener Stadterweiterungsamt (1909-1914) unter dem Bau-Beigeordneten Robert Schmidt, der in jenen Jahren die Gründung des Siedlungsverbands Ruhrkohlenbezirk vorbereitete - eine der ersten deutschen Raumplanungsbehörden - und in Berlin, wo er 1914 und 1918-1926 in der Verwaltung Groß-Berlins die Bauordnung und den Wirtschaftsplan für die Gesamtgemeinde aufstellte, folgte Heiligenthal 1927 dem Ruf an die Technische Hochschule Karlsruhe, wo er sich bis zu seiner Emeritierung 1949 vor allem als Wegbereiter der Landesplanung im Oberrheingebiet einen Namen machte. Die Ergebnisse der von ihm und seinen Mitarbeitern am Institut für Städtebau, Stadtwirtschaft und Siedlung gemachten Untersuchungen veröffentlichte er in den zwölf Heften der zwischen 1933 und 1941 erschienenen Siedlungsstudien ${ }^{(16)}$.

16 Für eine kritische Würdigung der Hefte 1-10 der Siedlungsstudien, s. Elsa Vonau, „À la recherche de l'unité perdue. Les travaux d'aménagement régional de Roman Heiligenthal 1933-1941“, Transeo, Nr. 2-3 („Figurer l'espace en sciences sociales“, April 2010), unter http://www.transeo-review.eu/Ala-recherche-de-1-unite-perdue.html (abgerufen am 12.5.2014). S. a. Christoph Bernhardt, André 
Trotz sich ideologisch anbiedernder Hefttitel wie Rasse und Wohnung in der großen Agglomeration blieb Heiligenthals Sicht auf den Oberrhein insgesamt die des Ökonomen, der hinter dem Verlust der politischen Einheit des Gebiets vor allem die verloren gegangene sozioökonomische Einheit bedauerte ${ }^{(17)}$. Im Zentrum seiner landesplanerischen Überlegungen stand daher in erster Linie der wirtschaftliche Aufschwung entlang des Flussraums, den er als Verkehrs- und Handelsverbindung zwischen Nord- und Südeuropa betrachtete. Besonders deutlich wird dies in den letzten beiden Bänden der Siedlungsstudien, die 1941 erschienen und dem wiedergewonnenen Elsass mit Straßburg gewidmet sind ${ }^{(18)}$. Parallel zum Planungswettbewerb "Groß-Straßburg“ von 1941, der die erzwungene Vision einer beiderseits des Rheins gelegenen Hauptstadt des aus Elsass und Baden gebildeten Oberrhein-Gaus heraufbeschwören sollte, veröffentlichte Heiligenthal eigene Vorschläge für die Neuordnung des Straßburger Großraums. Zwar legte auch er, wie in der Auslobung gefordert, ein monumentales politisches Forum, das die deutsche Herrschaft markieren sollte, in das Hafengebiet zwischen Straßburg und Kehl. Wichtiger sind aber seine Überlegungen zur Neuordnung des Straßburger Wirtschafts- und Verkehrsraumes beiderseits des Rheins. Denn diese nehmen die mit der Gründung der Communauté Urbaine de Strasbourg („Gemeindeverband Straßburg“) 1967 angestoßene und mit der Schaffung des Eurodistrikts Strasbourg-Ortenau 2005 forcierte Entstehung einer rheinübergreifenden "Eurometropole“ - so nennt sich der Gemeindeverband seit 2015 - vorweg.

Heiligenthal untersucht die Entwicklung Straßburgs und zwölf weiterer Rheinstädte von Schaffhausen bis Köln, vergleicht die Stadt mit Frankfurt; beide Städte sind seines Erachtens durch ihre geografische Lage an einem Rheinnebenfluss und ihre Verkehrslage in Bezug auf Rohstofflager miteinander verwandt. Heiligenthal hinterfragt auch die zukünftige Rolle des Straßburg gegenüberliegenden Rheinufers und betrachtet als mögliches Vorbild für die Entwicklung eines rheinübergreifenden Straßburger Großraums die Region Rhein-Neckar um Mannheim, Ludwigshafen und Heidelberg, deren Entwicklung ebenfalls durch die Grenznähe gestört war. Dabei fragt er sich, ob „Kehl lediglich die Rolle eines Brückenkopfes spielen wird, oder ob hier eine Stadt entstehen kann, etwa von der Größe und Bedeutung Ludwigshafens“(19) (Abb. 7).

In Karlsruhe wiederum werden Heiligenthals Kollegen, der Architekt und Stadtplaner Otto Ernst Schweizer und der Verkehrsplaner Friedrich Raab, 1943 vom Stadtoberhaupt mit Planungen für den Wiederaufbau und die zukünftige Entwicklung Karlsruhes betraut, die auch als Kompensationsmaßnahmen für den Verlust des

Guillerme, Elsa Vonau, L'émergence des politiques de développement durable dans un contexte transfrontalier: L'exemple du Rhin supérieur (1914-200o), Forschungsendbericht (Forschungsprogramm „Paysages et développement durable“ des MEDD), Erkner, Leibniz-Institut IRS u. Paris, CDHTECNAM, 2009.

17 Roman Heiligenthal, Rasse und Wohnung in der großen Agglomeration, Heidelberg, Carl Winter's Universitätsbuchhandlung (Siedlungsstudien, 8), 1937.

18 Roman Heiligenthal, Neubau Straßburgs. Grundlagen und Vorschläge, Heidelberg, Carl Winter's Universitätsbuchhandlung (Siedlungsstudien, 11), 1941; DERS., Straßburg unter den Rheinstädten, Heidelberg, Carl Winter's Universitätsbuchhandlung (Siedlungsstudien, 12), 1941.

19 R. Heiligenthal, Straßburg (Anm. 18), S. 5. 


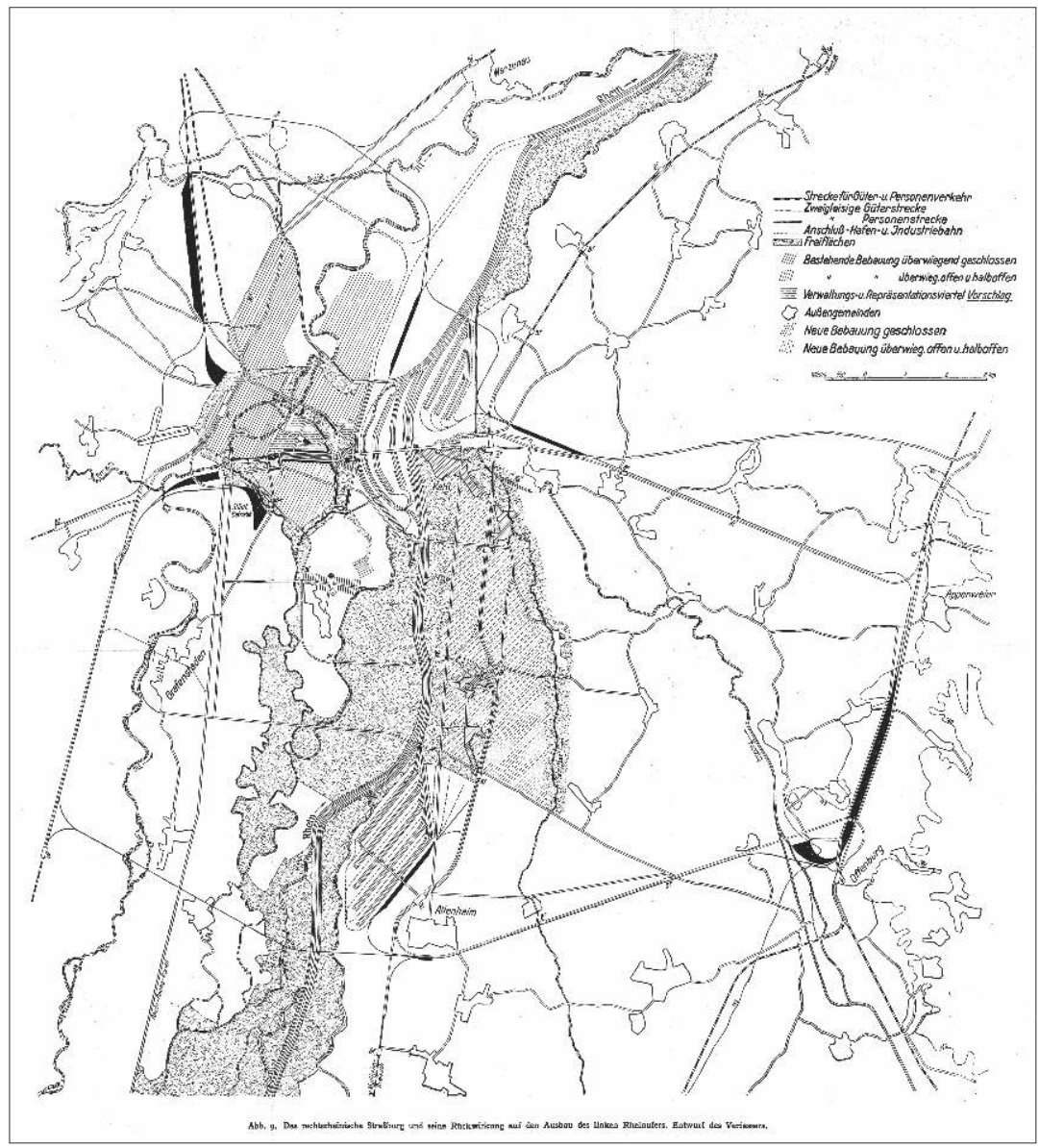

Abb. 7 - Plan zur Neuordnung des Großraums Straßburg,

Entwurf von Roman Heiligenthal (1941), in: Ders., Straßburg (Anm. 18), Abb. 9.

Status als Gauhauptstadt an Straßburg gedacht sind ${ }^{(20)}$. Der Geografie des Rheintals um Karlsruhe passt Schweizer seinen „Idealplan einer Großstadt“ von 1931 an, der Le Corbusiers starrem Schema der Ville contemporaine eine erweiterungsfähige „elastische“ Stadtstruktur entgegensetzt, die Parallelen mit Nikolai A. Miljutins Bandstadt aufweist. Dabei baut er bestehende Gemeinden mit neuen Wohnvierteln zu Trabantenstädten aus, die den Bezug zur Landschaft suchen und zusammen mit den geplanten Industrieanlagen mittels effizienter Verkehrsadern an die zum „Idealzentrum“ wiederaufgebaute Karlsruher Innenstadt gekettet werden (Abb. 8).

Im heutigen Karlsruher Stadtbahnsystem, dem auch in Frankreich und besonders in Straßburg beachteten „Karlsruher Modell“, sind von dieser Planung noch Anklänge zu finden.

20 Immo Boyken, Otto Ernst Schweizer 1890-1965. Bauten und Projekte, Stuttgart, Axel Menges, 1996, hier S. 150-153 u. 180-181. 


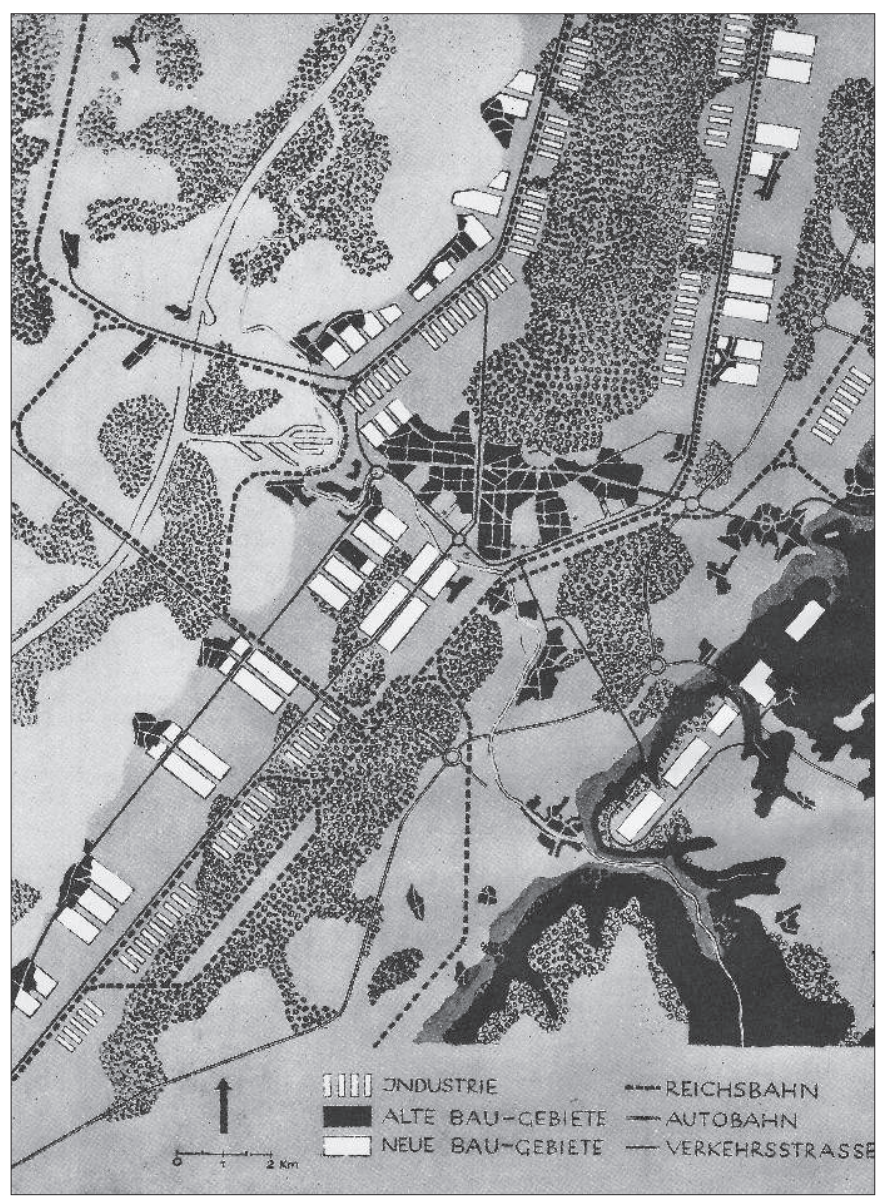

Abb. 8

Plan zur Neuordnung des Großraums Karlsruhe, Entwurf von Otto Ernst Schweizer und Friedrich Raab (1943-1944), in: W. Streif (Red.), Otto Ernst Schweizer (Anm. 21), S. 54.

Als Professor für Städtischen Hochbau, Wohnungs- und Siedlungswesen an der Technischen Hochschule Karlsruhe beschäftigte sich Schweizer ab 1930 ähnlich wie Heiligenthal mit Siedlungsfragen in der Region. Schweizers Ansatz ist jedoch nicht der eines Raumplaners; als Städtebauer löst er die Frage der Stadt-LandBeziehung im Konzept einer in Trabanten und selbständigen Nachbarschaften aufgelösten Siedlungsform auf.

$\mathrm{Zu}$ diesem Thema stellte Schweizer seit den 1930er Jahren immer wieder am Oberrhein verortete Entwurfsaufgaben und prägte als Verfechter eines funktionalistischen Städtebaus bis zu seiner Emeritierung 1960 eine ganze Generation von Architekten und Planern, die bis weit in die achtziger Jahre vor allem im Südwesten Deutschlands tätig waren $^{(21)}$. So wird die Städtebaulehre nach Schweizer durch seinen Schüler und Nachfolger Adolf Bayer vertreten, der sie zur Regionalplanung hin erweitert, und ab 1981 durch Martin Einsele und Klaus Richrath, letzterer ebenfalls ein ehemaliger Schüler Schweizers und Mitglied der Arbeitsgruppe Oberrhein um Einsele.

21 Otto Ernst SchweIzer, Über die Grundlagen architektonischen Schaffens. Mit Arbeiten von Studierenden der Technischen Hochschule Karlsruhe aus den Jahren 1930/34, Stuttgart, Julius Hoffmann, 1935; Werner STREIF (Red.), Otto Ernst Schweizer und seine Schule. Die Schüler zum sechzigsten Geburtstag ihres Meisters, Ravensburg, Otto Maier, 1950; Otto Ernst SchweIzer, Forschung und Lehre. 1930-1960, Stuttgart, Karl Krämer, 1962; Klaus Richrath (Hg.), Assistenten und Mitarbeiter von Professor Dr.-Ing. E.h. Otto Ernst Schweizer. Erinnerungen, Episoden, Interpretationen, eigene Arbeiten, Karlsruhe, Ernst Grässer, 2005. 
Während Heiligenthals und Schweizers Wirken am Oberrhein nur auf der deutschen Stromseite tiefe Spuren hinterließ, wurde die Arbeit des deutschen Geografen Walter Christaller zur Siedlungsstruktur und Regionalplanung am Oberrhein durchaus auch in Frankreich rezipiert. Christaller hatte Anfang der dreißiger Jahre seine „Theorie der zentralen Orte" aufgestellt, um die Regelmäßigkeit der Siedlungsverteilung im süddeutschen Raum zu erklären, wobei er neben der Nordwestschweiz ganz selbstverständlich auch Elsass-Lothringen in seine Betrachtung miteinbezog und bestehende Staats- und Landesgrenzen verwischte ${ }^{(22)}$. Denn nach seiner Auffassung besteht die Aufgabe der Landesplanung nicht darin, Regionen zunächst gegeneinander abzugrenzen und diese Gebiete dann der Planung zuzuführen. Stattdessen soll die Landesplanung das Einzugsgebiet der „Marktfunktion“ - gemeint sind Güter und Dienstleistungen jeder Art - einer gegebenen Stadt als ihr zentrales sozioökonomisches Kennzeichen betrachten, um aufbauend auf das Beziehungsnetz von Märkten und Einzugsgebieten einen kohärenten Raum zu definieren. Das Christallersche Modell ist sehr stark durchhierarchisiert; zentrale Einrichtungen und Dienstleistungen werden nach Wichtigkeit geordnet und Orten entsprechender Bedeutung (Ober-, Mittel-, Unter-, Kleinzentrum) zugeordnet, die über ein System von ebenfalls hierarchisierten Entwicklungsachsen miteinander verbunden sind. Orte einer niedrigeren Ordnung liegen dementsprechend immer

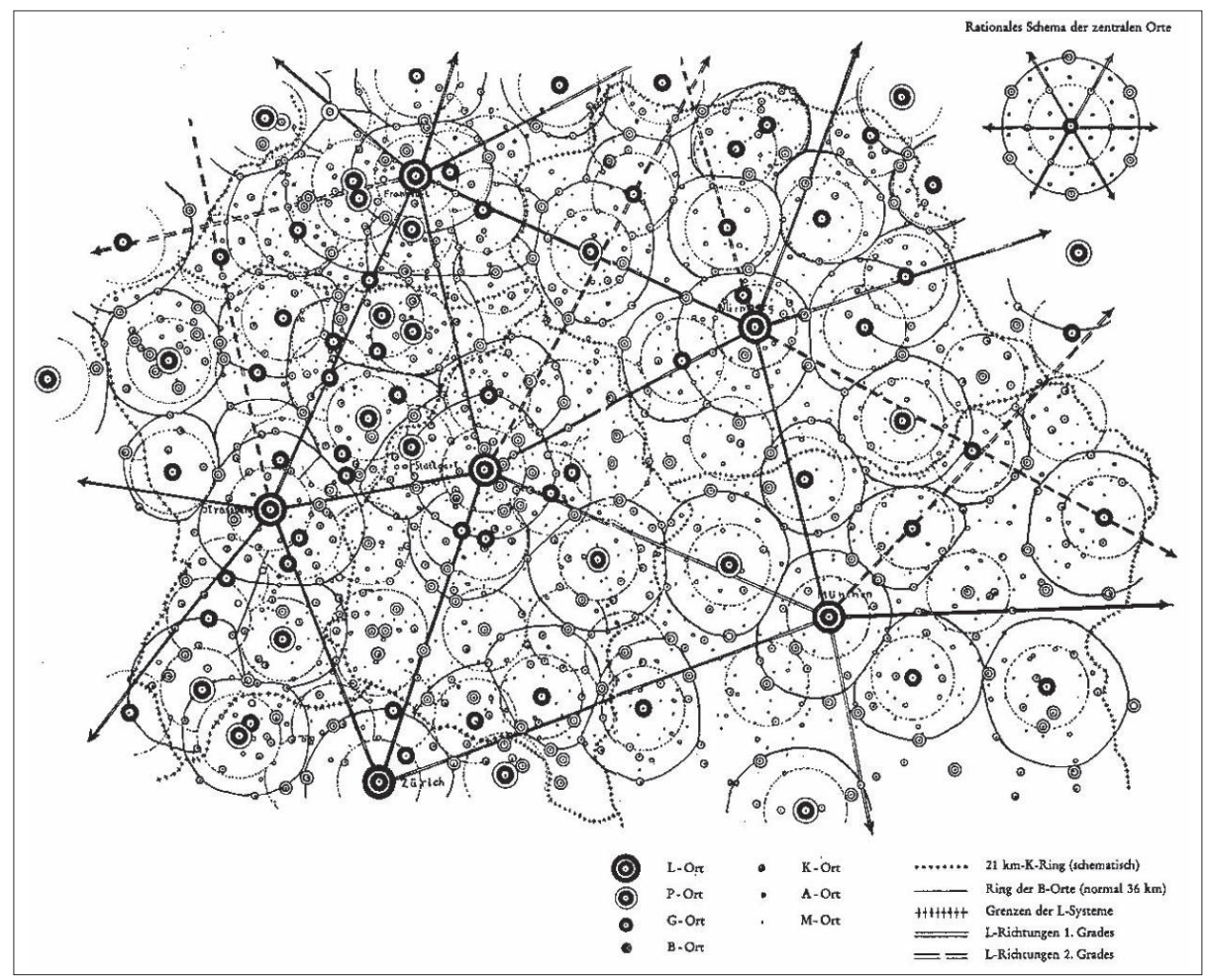

Abb. 9 - Das System der zentralen Orte in Süddeutschland, in: W. Christaller, Die zentralen Orte (Anm. 22), Karte 4. 
im Einflussbereich von Orten höherer Ordnung, welche zentrale Dienstleistungen für ihr Einzugsgebiet erfüllen und einen entsprechenden Bedeutungsüberschuss aufweisen. Für das erweiterte Oberrheingebiet sind laut Christaller die wichtigsten zentralen Orte Frankfurt, Straßburg, Stuttgart und Zürich (Abb. 9).

Christallers Theorie postuliert ein räumliches Organisationsschema, das direkt in die pyramidale Raumplanung und Siedlungspolitik des NS-Regimes einfloss und auch bis in die jüngste Zeit den Hintergrund der Raumordnungskonzepte zur Regional- und Landesplanung in Deutschland bildete, wo es, basierend auf dem Recht jedes Bürgers auf vergleichbare Lebensqualität, nunmehr den gerechten Zugang zu öffentlichen Einrichtungen und Dienstleistungen gewährleisten sollte. Auch in Frankreich gehörten Christallers Thesen zum Rüstzeug der Geografen und Raumplaner. So passt Michel Rochefort 1960 in seiner Dissertation zur Organisation urbaine de l'Alsace das Christallersche Ordnungssystem an die Gegebenheiten des Elsass an ${ }^{(23)}$. Für das französische Bauministerium leitet Rochefort in den Folgejahren die Untersuchungen zur armature urbaine Frankreichs und seiner Regionen. Um dem Entwicklungsgefälle in der urbanen Siedlungsstruktur zwischen Paris und Provinz entgegenzuwirken, fordert die Arbeitsgruppe um Rochefort unter anderem die Schaffung von acht métropoles d'équilibre im Land, was 1967 auch die Gründung des Gemeindeverbands Straßburg nach sich zieht ${ }^{(24)}$. Zur selben Zeit entwickelt die elsässische Raumplanungsstelle ein Schema zur regionalen Siedlungsstruktur des Elsass, das sich auf Rocheforts Arbeiten und auf bei Christaller entlehnte geografische Begriffe stützt ${ }^{(25)}$ (Abb. 10).

Die Pertinenz dieses Systems mit seinem starren Zentralitätsbegriff steht angesichts der Dynamik heutiger gesellschaftlicher und ökonomischer Veränderungsprozesse spätestens seit den neunziger Jahren zur Debatte. Die Karten zur „dezentralen Dreiländer-Metropole Oberrhein“ von 1999 bilden genau diesen Übergang von einem wirtschaftsorientierten System der „Hierarchie der Zentren“ zu einem „Städtenetz in einer Gartenlandschaft“ ab, dessen Entwicklung nachhaltig gestaltet werden soll ${ }^{(26)}$. Dies mündet in die Forderung nach mehr governance und weniger Hierarchie in Politik und Planungskultur sowie nach einer Aufwertung der Kulturlandschaft am Rhein, die der Verstädterung bzw. der Metropolenentwicklung regulierend entgegensetzt werden soll. Nicht über ein urbanes Kontinuum, sondern durch regionale Verkehrsverbünde soll die „Dreiländer-Metropole Oberrhein“ zusammenwachsen.

Die Entwicklung der Mobilität stand auch im Vordergrund einer französischen Studie des staatlichen Instituts für Verkehrsforschung INRETS aus dem Jahre 1992, in der drei Zukunftsszenarien aufgezeigt wurden. Dem „kalifornischen Szenario“ einer

23 Michel Rochefort, L'organisation urbaine de l'Alsace, Paris, Les Belles Lettres, 1960. S. a. Roland Schwab, Jean-Paul Martin, „Méthodes d'analyse de l'armature urbaine régionale“, Revue Géographique de l'Est, Nr. 1-2 (Januar-Juni 1968), S. 57-87.

24 Michel Rochefort, Jean Hautreux, La fonction régionale dans l'armature urbaine française (Bericht für das französische Bauministerium), Paris, Ministère de la Construction, 1963-1964. S. a. Dominique Rivière, Bernard Bret, „Michel Rochefort, pour une géographie urbaine engagée“, Confins, Nr. 23 (2015), unter http://confins.revues.org/10140 (abgerufen am 20.6.2015).

25 DRE AlsAcE, Rapport sur les perspectives de développement des villes d'Alsace, Circonscription d'action régionale d'Alsace, Straßburg, Direction Régionale de l'Équipement d'Alsace, Oktober 1965.

26 Dt.-frz.-schw. Oberrheinkonferenz (Hg.), Lebensraum Oberrhein (Anm. 10), S. 140-141 u. 146-147. 


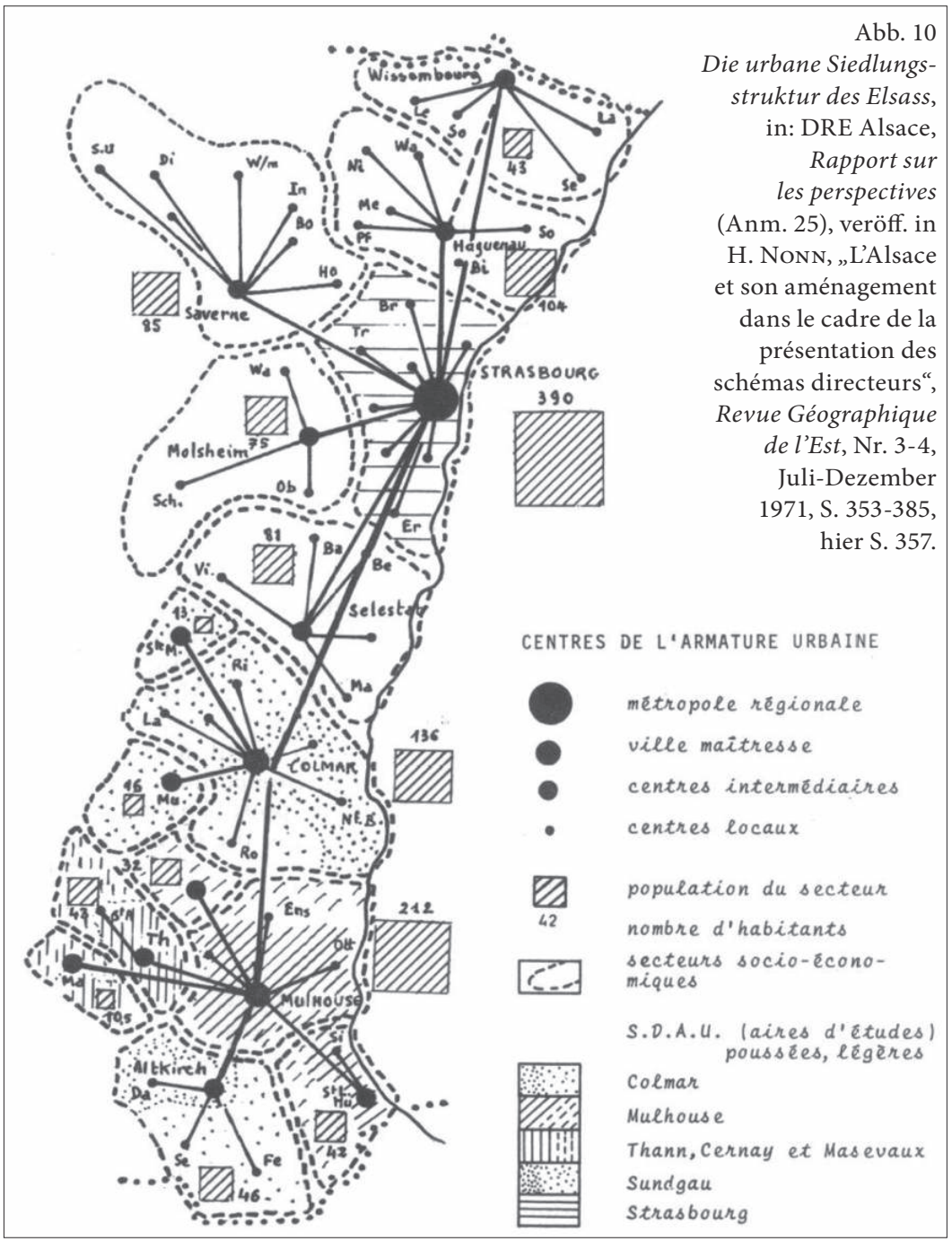

rein am ökonomischen Liberalismus orientierten, diffusen und automobilorientierten Stadtentwicklung wird das „saint-simonistische Szenario“ einer staatlich gelenkten Entwicklung gegenübergestellt, die sich wie im Falle des Großraums Paris auf die Millionenstadt konzentriert und die Verkehrsnetze entsprechend ausrichtet. Als dritter Weg wird der Oberrhein diskutiert ${ }^{(27)}$. Demnach zeichnet sich das „rheinische Szenario" durch eine munizipalistische, das heißt von einer reformorientierten Kommunalpolitik getragenen Stadtentwicklung aus, die sich auf den öffentlichen Nahverkehr, alternative Mobilitätskonzepte und innovativen Wohnungsbau stützt - ausgehend

27 Alain Biber, Marie-Hélène Masson, Jean-Pierre Orfeuil, Questions vives pour une prospective de la mobilité quotidienne (Synthèse INRETS, 19), Arcueil, INRETS (Institut national de recherche sur les transports et leur sécurité), 1993. 
von den Gartenstädten des beginnenden 20. Jahrhunderts bis zu den nachhaltigen Stadtvierteln in unserer Zeit.

Auf diese Studie bezog sich auch der elsässische think tank Association de Prospective Rhénane (APR), der in einem 1996 dem Regionalrat Elsass vorgelegten Bericht noch einen Schritt weiter geht und von einem modèle rhénan der Raum- und Siedlungsstruktur spricht ${ }^{(28)}$. Allerdings ist der Begriff schwer zu fassen, da er ähnlich wie in Haus Oberrhein-Panorama neben geografischen auch sozioökonomische und (kultur) geschichtliche Aspekte konnotiert. Zudem ist das modèle rhénan in stetem Wandel begriffen. So plädiert der elsässische Geograf Henri Nonn, einer der Gründer der APR, in seinen Studien zur Geografie des Elsass für eine dynamische Betrachtung des Oberrheins, der wie jede andere Region Veränderungen unterworfen ist, die ihn immer wieder neu bestimmen und in denen sich verschiedenste Einflüsse überlagern ${ }^{(29)}$.

In Deutschland verbindet man mit dem Begriff „,rheinisches Modell“ den rheinischen Kapitalismus, also die "gute alte“ soziale Marktwirtschaft der Bonner Republik, während das Schlagwort modèle rhénan in Frankreich mehrdeutig ist, da es über das Wirtschaftsmodell hinaus auch ein geografisches Erklärungsmodell für eine von drei Formen der Regionalstruktur und -entwicklung in Westeuropa bezeichnet. Diese Fassung des Begriffs geht auf die Studien des Geografen Étienne Juillard zurück, der als Professor für Geografie und Raumplanung (1954-73) sowie als Gründer und Leiter (1959-73) des Centre de Recherches Régionales Nonns Mentor und Vorgänger an der Straßburger Universität Louis Pasteur war. Juillard, der im Elsass aufwuchs und kurzzeitig auch in Nancy lehrte, befasste sich als Forscher, Universitätsprofessor und Politikberater von der unmittelbaren Nachkriegszeit bis in die siebziger Jahre mit Fragen der Regionalentwicklung, insbesondere des Elsass. In seinem 1968 erschienenen Werk L'Europe rhénane beschreibt er anhand der Geschichte und Geografie der Gebiete und Regionen zwischen Alpen und Nordsee ein „rheinisches Europa“ als dicht besiedelten, polyzentrischen und vielfältig verbundenen Kultur- und Wirtschaftsraum mit dem Fluss als Lebensader ${ }^{(30)}$. Im Unterschied zu den Weltstädten, deren „Gigantismus“ das Entstehen verfeinerter Zivilisationsformen begünstigt habe, blieben die „rheinischen Metropolen“ (ZürichBasel, das Rhein-Main-Gebiet, die Region Rhein-Ruhr und die Randstad) „immer noch Städtegebilde im menschlichen Maßstab, in denen ein maßvoller Städtebau darauf hinzuwirken scheint, die Lebensart des 21. Jahrhunderts hervorzubringen“(31).

Eindeutige Zukunftsperspektiven für diese „rheinische Zivilisation“ sieht Juillard mit der Dekolonisation und dem EG-Fusionsvertrag (1967) gegeben, deren Folge eine bessere Innenentwicklung des Kontinents um die sechs Gründerstaaten der EG sein könne, mit dem „einenden Rhein“ als europäische Entwicklungsachse von Antwerpen und Rotterdam bis über die Alpen zu den lombardischen Städten ${ }^{(32)}$. Juillards Verdienst ist es also, die Arbeit zur Entwicklung und zur Problematik der Gebiete am Rhein in

28 Association de Prospective Rhénane, Éléments d'analyse sur le modèle rhénan d'organisation urbaine (Schlussbericht für den Regionalrat Elsass), Straßburg, Association de prospective rhénane, 1996.

29 Henri Nonn, L'Alsace et ses territoires, Straßburg, Presses universitaires de Strasbourg, 2008.

30 Étienne Juillard, L’Europe rhénane. Géographie d'un grand espace, Paris, Armand Colin, 1968.

31 Ebd., S. 262.

32 Ebd., S. 272-274. 

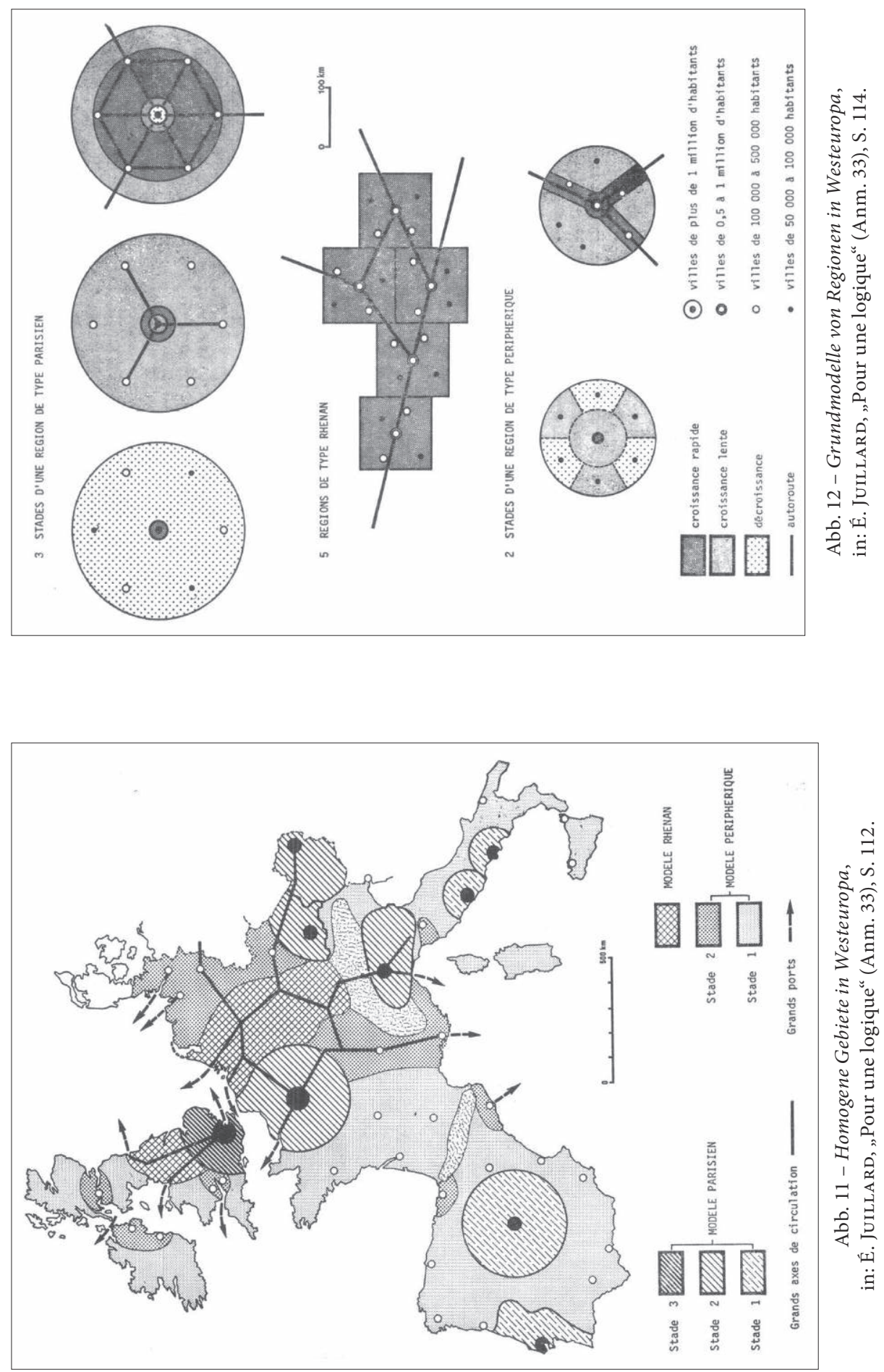
einen europäischen Kontext zu stellen, was uns heute so selbstverständlich erscheint, vor fünfzig Jahren aber durchaus ein Akt der Befreiung war. Denn damit überwindet er sowohl die von hegemonialen Begehrlichkeiten durchtränkte Sicht mancher deutscher Geografen auf den (deutschen) Kulturraum im Einzugsgebiet von „Vater Rhein und seinen Töchtern" wie auch die jahrhundertelang verfolgte französische Forderung nach der Rheingrenze.

Gemeinsam mit Nonn führt Juillard am Centre de Recherches Régionales Anfang der siebziger Jahre seine Überlegungen in einer Vergleichsstudie zur regionalen Raumgliederung in Westeuropa fort. Die Analyse verschiedener Kriterien wie Städteverteilung, Siedlungsdichte, Wirtschaftskraft und Verkehrsinfrastruktur ergibt eine Klassifizierung in drei raumordnerische Strukturtypen: das modèle rhénan mit seiner polyzentrischen Siedlungsstruktur, das auf eine Zentralstadt fixierte modèle parisien und das dezentrale bzw. ländliche modèle périphérique ${ }^{(33)}$. Die Strukturtheorie differenziert letztere jeweils noch in verschiedene Entwicklungsstufen, wohingegen das modèle rhénan als stabile oder - wie man heute sagen würde - nachhaltige Siedlungsstruktur dargestellt wird. Die dem modèle rhénan zugerechneten Gebiete reichen von den Industriezentren der englischen Midlands über die Nordsee und Schweizer Mittelland verbindende Rheinschiene bis über die Alpen zum lombardischen Städtenetz in der Po-Ebene (Abb. $11+12$ ).

Sie sind fast deckungsgleich mit der sogenannten „Blauen Banane“, dem einprägsamen Bild einer „europäischen Megalopolis“, die als blauer Streifen die Europäische Union durchzieht. Auch für diesen 1988 geprägten Begriff steht mit Roger Brunet ein französischer Geograf Pate. Auf der Basis von Juillards Thesen betrachtete Brunet in seinen Untersuchungen zum Wirtschaftspotential (West-)Europas den europäischen Großraum zwischen Südengland und Norditalien als eine Kette urbanisierter Regionen mit überdurchschnittlichem Wirtschaftswachstum und als Motor des europäischen Integrationsprozesses ${ }^{(34)}$. Die Darstellung war auch als Aufforderung an die französische Regierung gerichtet, der Marginalisierung von Paris, das außerhalb der „europäischen Megalopolis“ liegt, entgegenzusteuern. Mittlerweile bekommt das Bild einer zentralen europäischen Entwicklungsachse durch den Oberrhein Konkurrenz durch jüngere Modelle von Entwicklungsachsen, -richtungen und -regionen. Genannt seien hier nur der "Atlantikbogen“ (Arc Atlantique), der „europäischer Sunbelt“ genannte Gürtel von Hightech-Regionen und das optimistische Bild einer „Traube Europa“, das eine Fülle von prosperierenden Metropolregionen und Entwicklungsstandorten in der erweiterten Europäischen Union zwischen Lissabon und Helsinki, Dublin und Athen zeigt ${ }^{(35)}$.

33 Étienne Juillard, Henri Nonn u. a., Espaces et régions en Europe occidentale, Éditions du CNRS (Reihe Actions thématiques programmées sciences humaines du CNRS, 10), 1976; Étienne JUILLARD, „Pour une logique des divisions régionales en Europe occidentale“, Revue Géographique de l'Est, Nr. 3-4 (Juli-Dezember 1976), S. 103-120.

34 Roger Brunet, La France dans l'espace européen, Montpellier, Reclus-GEM Régions, 1988.

35 Guy Baudelle, „Figures d'Europe : une question d'image(s)“, Norois, Nr. 194 (2005/1), unter http:// norois.revues.org/604 (abgerufen am 13.10.2014). 


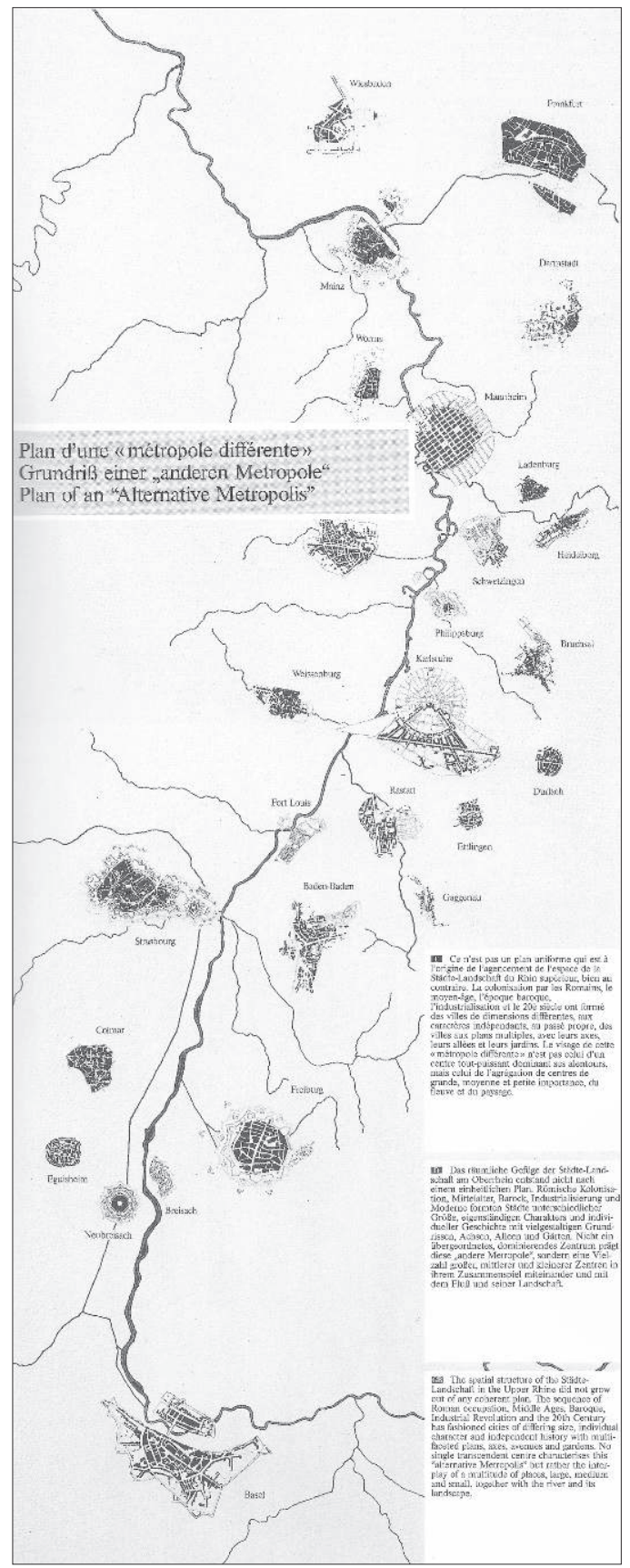

Abb. 13

Grundriss einer „anderen Metropole“, in: M. EINSELE (Hg.), Le Rhin supérieur (Anm. 8), S. 33.

\section{Zusammen wachsen und zusammenwachsen}

So lautet das Motto der trinationalen IBA Basel 2020. Wiewohl die IBAs (Internationale Bauausstellungen) auch eine sehr deutsche Form der Bauausstellung sind, wird sie in diesem Fall von einer Stadt des Nicht-EU-Mitglieds Schweiz getragen und erstmals auch französische Kommunen miteinbeziehen. Das bedeutet auch, dass das Fallen der Staatsgrenzen innerhalb der Europäischen Union zwar ein wichtiger, aber bei weitem nicht der einzige Faktor für einen „vereinten“ Oberrhein ist. Austausch, Transfer und Interferenzen hat es in dieser Region trotz wechselnder Grenzen und Zugehörigkeiten immer gegeben ${ }^{(36)}$. Im 19. Jahrhundert und noch bis 1918 wurden Generationen von Architekten aus dem Elsass, aus Baden und aus Lothringen gleichermaßen an den Technischen Hochschulen in Karlsruhe und Stuttgart, an der École des Beaux-Arts in Paris oder am Eidgenössischen Polytechnikum in Zürich ausgebildet. Ihr Einfluss auf die Architektur und die Stadtgestalt beiderseits des Rheins war bis in die fünfziger Jahre spürbar, ungeachtet der beiden Weltkriege. Es folgten zwei Jahrzehnte relativen Desinteresses und Misstrauens gegenüber dem Nachbarland, aber auch hinsichtlich des gemeinsamen baulichen und kulturellen Erbes. Die

36 Jean-Louis Cohen, Hartmut Frank (Hg.), Interferenzen / Interférences. Deutschland-Frankreich, Architektur 1800-2000, Ausst.-Kat., Tübingen/Berlin, Wasmuth, 2013 (Frz. Ausg.: Interférences / Interferenzen. Architecture, Allemagne-France, 1800-2000, Straßburg, Éd. des Musées de Strasbourg, 2013). 
Vorstellung des Oberrheins als grenzübergreifendem Kultur- und Lebensraum kam zwar in den siebziger Jahren wieder auf, doch wirklich durchsetzen konnte sie sich erst mit der Wertschätzung, die das architektonische und städtebauliche Erbe aus der sogenannten „deutschen“ Periode im Elsass und in Lothringen in jüngster Zeit erfährt. Dazu gehören auch die laufenden Vorbereitungen Straßburgs zur Anmeldung der nach der Annexion von 1871 errichteten Neustadt als Unesco-Weltkulturerbe (Abb. 13).

Im Bereich von Stadt- und Regionalplanung kann man erst am Ende des 20. Jahrhunderts von grenzüberschreitender Zusammenarbeit reden - da gehörte die tägliche Fahrt über den Rhein längst zum Alltag von Tausenden von Grenzgängern. Insofern gingen die ersten Gemeinschaftsprojekte nicht etwa wichtigen Ereignissen und Wandlungsprozessen voraus, sondern sind vielmehr ihr Ergebnis. Konkret entstanden sie als Folge der gemeinsamen Anstrengungen zum Schutz der Region vor Störfällen in Anlagen mit Risikopotential. Ab 1971 haben sich elsässische und badische Bürgerinitiativen erfolgreich gegen den Bau eines Kernkraftwerks im badischen Wyhl gewehrt und kämpften gemeinsam für die Schließung des Atommeilers im elsässischen Fessenheim. Nach der Umweltkatastrophe von Schweizerhalle - ausgelöst durch den Chemieunfall in einem Industriegebiet bei Basel im Jahr 1986 - wurde die Kooperation zwischen den Anrainerstaaten des Rheins, die bis dahin im Kielwasser der Bürgerbewegung verblieben war, auch formell erweitert. Eine dauerhafte Zusammenarbeit zwischen Straßburg und dem rechtsrheinischen Kehl entstand Ende der achtziger Jahre. Sie fand ihren Ausdruck im gemeinsamen Kampf beider Städte gegen den Bau einer Müllverbrennungsanlage, die das Land Baden-Württemberg am äußersten Rand seiner Landesgrenzen errichten wollte. Die westliche Rheinseite schien auf den Landkarten jener Zeit noch ein weißer Fleck zu sein.

Seit der Vollendung des Europäischen Binnenmarkts, insbesondere infolge der gegenseitigen Anerkennung von Bildungsabschlüssen und der Niederlassungsfreiheit, sind die Städte und Gemeinden am Oberrhein darauf angewiesen, sich zu vernetzen, etwa um sich dem Problem der Zersiedelung der Landschaft zu stellen. Gemeinsam wird die Entwicklung ländlicher Kommunen beiderseits des Rheins verfolgt und ihre Flächenpolitik verglichen, grenzüberschreitende Metropolräume werden gebildet, von den deutsch-französischen Eurodistrikten Regio PAMINA um Karlsruhe, Strasbourg-Ortenau und Region Freiburg / Centre et Sud Alsace bis zum Trinationalen Eurodistrikt Basel.

In einem von Standortwettbewerb und city branding gekennzeichneten aktuellen Kontext kommt auch Straßburg nicht umhin, die Vision seiner Entwicklung neu zu überdenken. Das betrifft vor allem die Beziehung zu anderen Agglomerationen am Oberrhein, die alle mit einem eigenen Image aufwarten, das ihre Identität als Metropolregion unterstreicht, und dies mit sehr unterschiedlichen Strategien verfolgen. So richtet der Trinationale Eurodistrikt Basel die IBA Basel 2020 aus, während sich die „TechnologieRegion Karlsruhe“ um die im Zuge der Exzellenzinitiative zum Karlsruher Institut für Technologie zusammengeschlossenen Universitäts- und Forschungseinrichtungen sowie eine Anzahl von Unternehmen und Labors aus dem Sektor der Spitzentechnologien konzentriert.

Basel, seit jeher Stadt am Rhein, beschreitet im Rahmen der IBA mit der Entwicklung des alle drei Staatsgrenzen und den Fluss überschreitenden Quartiers „3Land“ neue Wege, aber auch Karlsruhe und Straßburg und möchten endlich an und über 


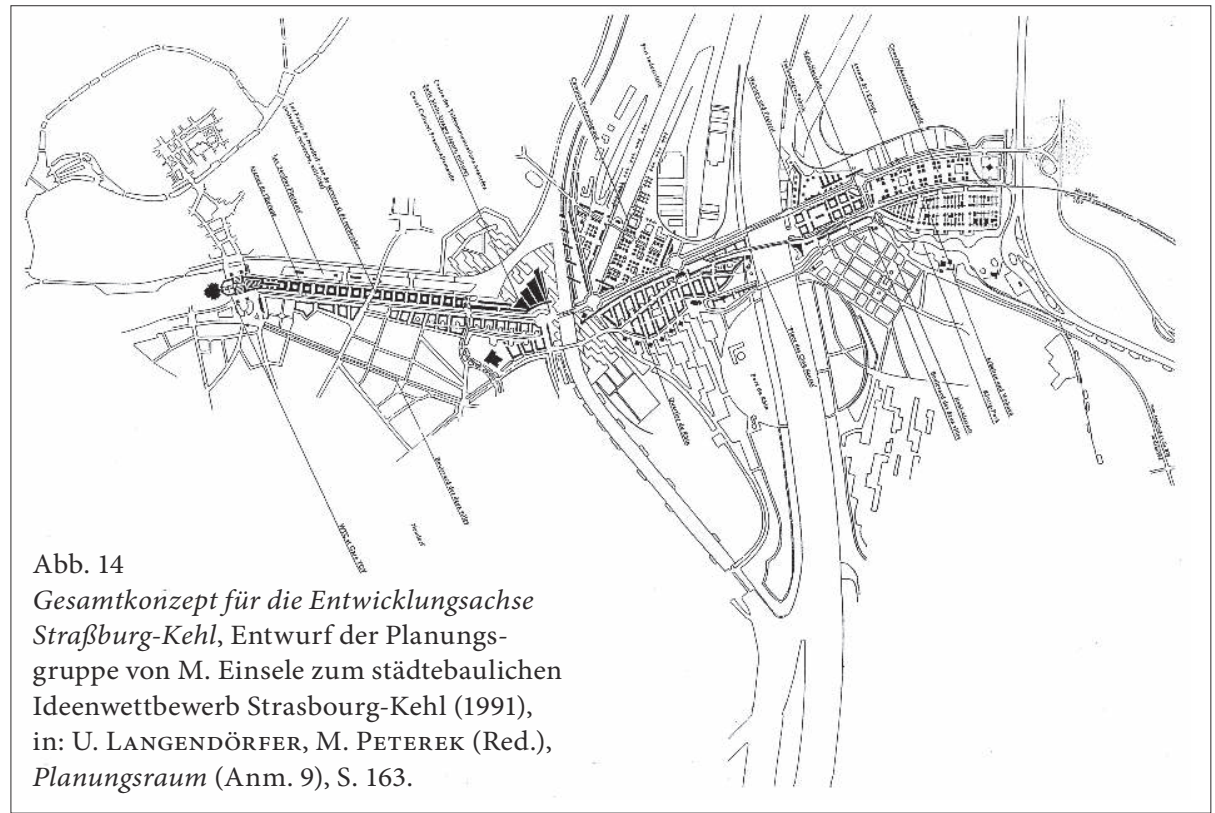

den Rhein wachsen. Unter dem Motto „Wie plant man eine Planstadt weiter?“ wurde im Hinblick auf das 300-jährige Stadtjubiläum 2015 ein räumliches Leitbild für Karlsruhe entwickelt, das die Fächerstadt in einem großen Stadtentwurf über Industrieund Hafenareale hinweg mit dem Rhein verbinden soll. Ein ähnliches Ziel verfolgt die Verbandsgemeinde Straßburg gemeinsam mit der Stadt Kehl schon seit geraumer Zeit, wobei der im Zuge der Landesgartenschau 2004 eingeweihte Garten der zwei

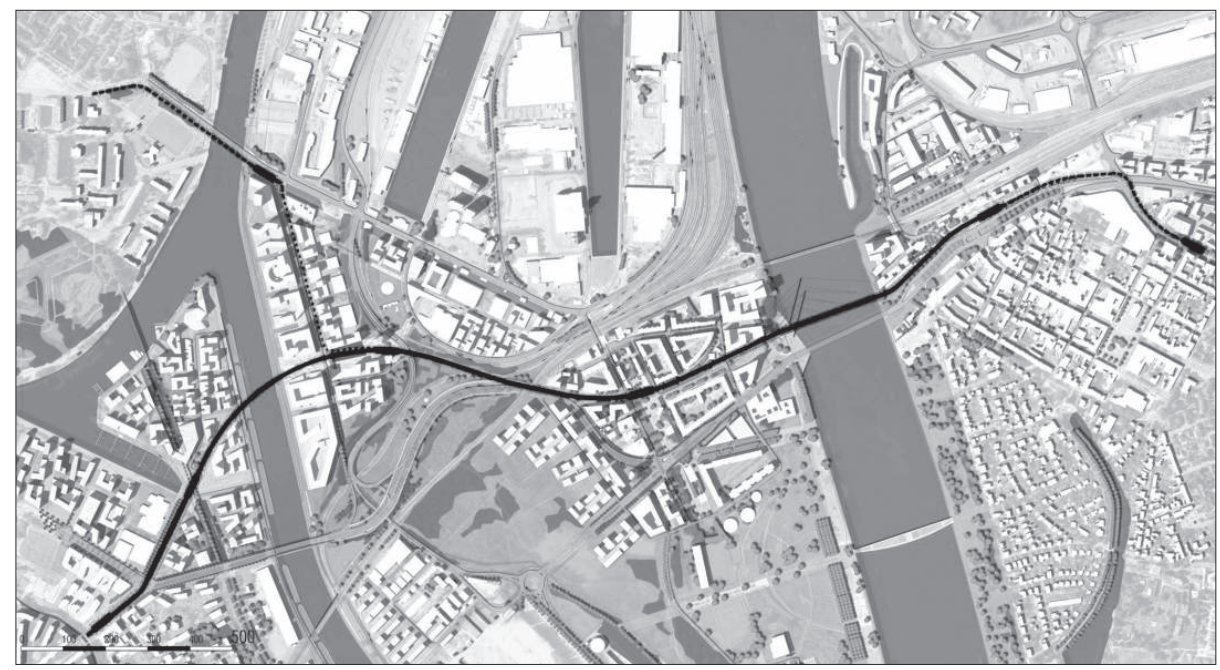

Abb. 15 - Masterplan für den Ostteil der Entwicklungsachse Straßburg-Kehl, 3D-Simulation zum Schéma directeur des Deux Rives von B. Reichen, A. Peter u. Partner (2011), Quelle: Communauté Urbaine de Strasbourg. 
Ufer mit seiner symbolträchtigen Fußgängerbrücke über den Fluss der erste Baustein des neuen, „rheinischen“ Straßburgs darstellt ${ }^{(37)}$. Welches sind die Merkmale der Baukultur der sich nunmehr „Eurometropole“ nennenden Verbandsgemeinde Straßburg? Eine nachhaltige Stadtentwicklung, die auf Konversion von Brachen entlang der Entwicklungsachse Straßburg-Kehl setzt, mit wichtigen öffentlichen Bauten und Freiräumen für die Kreativwirtschaft, mit nachhaltigen Stadtquartieren und mit Baufeldern für Baugruppen - beispielgebend sind hier Tübingen und Freiburg - mit alternativen Mobilitätsnetzen und einer Straßenbahnlinie über den Rhein. Ein besonderes Gewicht liegt dabei auf der Entwicklung eines flussübergreifenden Quartiers, dem eine symbolische Bedeutung zukommt, das aber auch alltagstauglich sein soll. Welche Urbanität wird für dieses komplexe Gebiet gewünscht? (Abb. 14)

Diese Frage sollte ein von der Verbandsgemeinde Straßburg und der Stadt Kehl Ende 2012 gemeinsam ausgelobter städtebaulicher Wettbewerb zu den Straßburger und Kehler Zollhofarealen beantworten, was aber durch die Wahl von zwei Preisträgern letztlich misslang. Den beiden Siegerentwürfen liegt nicht nur ein unterschiedliches Verständnis von Urbanität zu Grunde, sondern auch von dem, was Planung leisten soll und kann. Eine gemeinsame Debatte über Stadtkultur und Planungskultur bleibt Straßburg und Kehl nicht erspart, wenn der Rhein zum identitätsstiftenden Raum der grenzüberschreitenden „Eurometropole“ werden soll. Sicherlich ist diese deutschfranzösische Zusammenarbeit fest in einem neuen Europa mit ähnlichen Lebensweisen auf beiden Seiten der Grenzen verankert, doch für welche Klientel wird neuer Wohnraum am Rhein geschaffen? Werden die neuen Stadtbausteine auch den Bedürfnissen der Bewohner der umliegenden Stadtteile gerecht und wie werden sie deren Leben verändern? Der Einzug der Bewohner in die neuen Stadtquartiere in Straßburg und der Zuzug französischer Familien in Kehl soll die Beziehung zum Rhein zugunsten einer neuen räumlichen Nähe und sich immer mehr vermischender Lebensformen verändern. Zwischen den Lebenswelten der Alteingesessenen und der Neuanwohner soll ein Gleichgewicht entstehen. Dazu muss ein neuer Blick auf die Gebiete zwischen Straßburg und Kehl geworfen werden, die heute zwar an den Rändern beider Städte liegen, aber morgen vielleicht im Herzen einer Metropole (Abb. 15).

\section{Verwendete Textpassagen:}

Im vorliegenden Beitrag hat der Verfasser Auszüge aus folgenden Texten verwendet:

Yves Gendron, Michel Messelis, Volker Ziegler, „Planen und bauen über den Rhein“, in: JeanLouis Cohen, Hartmut Frank (Hg.), Interferenzen (Anm. 36), S. 426-431 (Frz. Ausg.: „Projeter par-dessus le Rhin“, in: Dies., Interférences (Anm. 36), S. 428-433).

Volker ZiegleR, „Straßburg wächst über den Rhein. Vom Grenzraum zur Städtelandschaft Oberrhein“, PlanerIn, Nr. 2 („Räumliche Leitbilder. Karlsruhe 300 Jahre nach dem Großen Plan“, April 2015), S. 11-13.

37 Weiterführende Literatur (chronologisch): Francis Cuillier (Hg.), Strasbourg. Chroniques d'urbanisme, Straßburg, ADEUS u. La Tour d’Aigues, Éditions de l'Aube, 1994; Yannis Tsiomis, Volker Ziegler, Anatomie de projets urbains. Bordeaux, Lyon, Rennes, Strasbourg, Paris, Éd. de la Villette, 2007; Cristiana Mazzoni, Luna D’Emilio (Hg.), Strasbourg métropole. Images et récits pour la villearchipel, Paris, La Commune, 2014. 


\section{Résumé}

Dans le cadre de la Triennale de Milan 1988, la vision d'une métropole du Rhin supérieur a été présentée à un public large et mise en relation avec les régions métropolitaines polycentriques de la Randstad et de la vallée du Pô en termes d'histoire (culturelle), de géographie, d'économie, etc. Remontant en partie aux années trente, les réflexions de géographes allemands et français comme Walter Christaller, Michel Rochefort, Étienne Juillard et Henri Nonn ainsi que les études et projets d'urbanisme et d'aménagement que Roman Heiligenthal, Otto Ernst Schweizer, professeurs d'urbanisme à Karlsruhe, et leurs disciples et successeurs ont mené dans la région du Rhin supérieur, forment l'outillage théorique et pratique pour aménager la métropole du Rhin supérieur. Toutefois, l'acceptation du terme métropolitain prend une connotation différente selon le contexte culturel; elle renvoie aux modèles de planification urbaine ou régionale de la Stadtlandschaft, côté allemand, et du "modèle rhénan ", côté français. Avec la chute des frontières au sein de l'Europe, l'axe rhénan s'impose comme moteur économique et principale artère de circulation de l'Union européenne. Toutefois, la nécessité d'embrayer vers un développement urbain durable et de protéger l'héritage matériel et immatériel des villes et paysages rhénans fait émerger des projets transfrontaliers supposés façonner les espaces de la vie de tous les jours au sein de la métropole du Rhin supérieur.

\section{Zusammenfassung}

Im Zuge der Triennale von Mailand 1988 wurde die Vision einer Metropole Oberrhein einem breiten Publikum vorgestellt und mit den polyzentrischen Metropolräumen der niederländischen Randstad sowie der Po-Ebene (kultur-)geschichtlich, geografisch, wirtschaftlich usw. in Bezug gesetzt. Zum Teil bis in die dreißiger Jahre zurückreichende Überlegungen deutscher und französischer Geografen wie Walter Christaller, Michel Rochefort, Étienne Juillard und Henri Nonn sowie Studien und Entwürfe der Karlsruher Städtebauer Roman Heiligenthal und Otto Ernst Schweizer sowie ihrer Schüler und Nachfolger zur Stadt- und Raumplanung am Oberrhein bilden dabei das theoretische und praktische Planungsrüstzeug für die Metropole Oberrhein. Allerdings ist der Metropolbegriff kulturspezifisch verschieden konnotiert und greift auf entsprechende stadt- bzw. raumplanerische Leitbilder zurück: auffranzösischer Seite auf das modèle rhénan, auf deutscher Seite auf die "Stadtlandschaft“. Mit dem Fallen der innereuropäischen Grenzen rückt einerseits die Rheinschiene als Wirtschaftsmotor und Hauptverkehrssachse der EU in den Vordergrund, andererseits mündet die Forderung nach einer nachhaltigen Stadtentwicklung und nach Schutz des Kultur- und Landschaftsraums am Rhein in grenzüberschreitende Planungen, die konkrete Lebensräume und Alltagswelten für die Metropole Oberrhein gestalten sollen. 